\title{
Finite dimensional Hamiltonian system related to Lax pair with symplectic and cyclic symmetries
}

\author{
Zi-Xiang Zhou \\ School of Mathematical Sciences, Fudan University, Shanghai 200433, China \\ E-mail: zxzhou@fudan.edu.cn
}

\begin{abstract}
For the $1+1$ dimensional Lax pair with a symplectic symmetry and cyclic symmetries, it is shown that there is a natural finite dimensional Hamiltonian system related to it by presenting a unified Lax matrix. The Liouville integrability of the derived finite dimensional Hamiltonian systems is proved in a unified way. Any solution of these Hamiltonian systems gives a solution of the original PDE. As an application, the two dimensional hyperbolic $C_{n}^{(1)}$ Toda equation is considered and the finite dimensional integrable Hamiltonian system related to it is obtained from the general results.
\end{abstract}

\section{Introduction}

There are many integrable nonlinear PDEs in $1+1$ dimensions [2, 22]. For quite a few of them, the related finite dimensional Liouville integrable Hamiltonian systems have been obtained. By this nonlinearization method [3, 4], the nonlinear PDE is changed to a system of nonlinear ODEs which are Liouville integrable Hamiltonian systems. Any solution of this system of nonlinear ODEs gives a solution of the original nonlinear PDE. This greatly simplifies the original problem. It is an effective way to obtain interesting exact solutions, especially quasi-periodic solutions of the nonlinear PDEs $[6,5,17,19,20,21]$. Soliton solutions can be obtained in this way by a limiting process [24]. Some integrable systems in higher dimensions have also been reduced to finite dimensional Liouville integrable Hamiltonian systems [6, 7, 8, 29, 30].

Usually these finite dimensional Hamiltonian systems have Lax matrices so that the Liouville integrability can be guaranteed $[9,10,18,25,26]$. Most results are

obtained for specific nonlinear PDEs and specific hierarchies with less symmetries, and the integrability of the derived finite dimensional Hamiltonian systems are proved case by case.

In the present paper, we consider a quite general Lax pair with a symplectic symmetry and cyclic symmetries. The Lax matrix is presented so that the nonlinear constraint of the lowest order is generated naturally from this Lax matrix. The Hamiltonian function for the ODEs derived from the nonlinear constraint is expressed in terms of the Lax matrix. The Liouville integrability of this Hamiltonian system 
is proved by obtaining the $r$ matrix and finding enough functionally independent conserved integrals. This system contains some known examples such as the MKdV equation and the nonlinear Schrödinger equation. It also contains any $n \times n$ AKNS system with $u(n)$ symmetry, where the symplectic structure is naturally derived from the complex structure, and the binary nonlinearization method [9] is recovered. As an application, the general results are used for the two dimensional $C_{n}^{(1)}$ hyperbolic Toda equation [12], one of the two dimensional affine Toda equations which are all integrable $[1,5,11,12,14,15,16,23]$. The two dimensional $C_{n}^{(1)}$ hyperbolic Toda equation has a natural symplectic structure. The finite dimensional Hamiltonian systems related to it are constructed explicitly. These Hamiltonian systems are simpler than (with space of lower dimension) that presented in [27] where binary nonlinear constraint was constructed. The result for the $x$-part of the Lax pair is derived from the general result of this paper, while that for the $t$-part which has the $\lambda^{-1}$ term is obtained independently.

The paper is organized as follows. In Section 2, some notations and the Lax pair with a symplectic symmetry and cyclic symmetries are presented. In Section 3, the Lax matrix and nonlinear constraint are obtained for this general system. The Hamiltonian function is also presented. The $r$ matrix is obtained in Section 4, which gives the involution of conserved integrals. The independence of the conserved integrals which are enough for Liouville integrability is proved in Section 5. In Section 6, the specific results, most of which are known, for the $2 \times 2$ real AKNS system, the MKdV equation, the nonlinear Schrödinger equation, the $u(n)$ AKNS system and the $n$ wave equation

are derived from the general conclusions. Finally, in Section 7, the results for the two dimensional $C_{n}^{(1)}$ Toda equation are derived.

\section{Notations and the Lax pair with symmetries}

Let $W$ be a $2 n \times 2 n$ invertible antisymmetric real matrix which gives a symplectic structure on $\mathbf{R}^{2 n}$.

Let

$$
G=\left\{A \in G L(2 n, \mathbf{C}) \mid A^{T} W A=W\right\},
$$

which is isomorphic to $S p(n, \mathbf{C})$, the complex symplectic algebra. The inner automorphism group of $G$ is $G /\{ \pm I\}$. Let $p: G \rightarrow G /\{ \pm I\}$ be the natural projection. Let

$$
\mathbf{g}=\left\{X \in g l(2 n, \mathbf{C}) \mid X^{T}=-W X W^{-1}\right\}
$$

be the Lie algebra of $G$.

Let $G_{0}$ be a finite subgroup of $G$ such that each of its element $A$ satisfies $\bar{A} A= \pm I$. Here $\bar{A}$ is the complex conjugation (without transpose) of $A$.

Lemma $1 p\left(G_{0}\right)$ is a finite Abelian subgroup of $G /\{ \pm I\}$. Therefore, for any $A, B \in G_{0}$, either $B A=A B$ or $B A=-A B$ holds. 
Finite dimensional Hamiltonian system related to Lax pair with symmetries

Proof: $\quad$ For any $A, B \in G_{0}, A B^{-1} \in G_{0}$. Hence $A^{-1} B A B^{-1}= \pm \overline{A B^{-1}} A B^{-1}=$ $\pm I$, which implies $B A= \pm A B$. The lemma is proved.

Suppose $\Omega_{1}, \cdots, \Omega_{N} \in G_{0}$ so that $p\left(\Omega_{a}\right)(a=1, \cdots, N)$ are generators of $p\left(G_{0}\right)$ and suppose the order of $p\left(\Omega_{a}\right) \in p\left(G_{0}\right)$ is $m_{a}$. Then, $\Omega_{a}$ 's satisfy

$$
\Omega_{a}^{T} W \Omega_{a}=W, \quad \bar{\Omega}_{a}= \pm \Omega_{a}^{-1}, \quad \Omega_{a}^{m_{a}}= \pm I .
$$

Let $\Sigma=\left\{\left(\alpha_{1}, \cdots, \alpha_{N}\right) \mid \alpha_{a} \in \mathbf{Z}(a=1, \cdots, N)\right\}, \Sigma_{0}=\left\{\alpha=\left(\alpha_{1}, \cdots, \alpha_{N}\right) \in \Sigma \mid 0 \leq\right.$ $\left.\alpha_{a}<m_{a}(a=1, \cdots, N)\right\}$, then we can write $\Omega^{\alpha}=\Omega_{1}^{\alpha_{1}} \cdots \Omega_{N}^{\alpha_{N}}$ etc. for multi-index $\alpha=\left(\alpha_{1}, \cdots, \alpha_{N}\right) \in \Sigma$. Denote $m_{0}$ to be the exponent of $p\left(G_{0}\right)$, which is the minimal common multiple of $m_{1}, \cdots, m_{N}$.

Let $\omega: G_{0} \rightarrow S^{1}=\{z \in \mathbf{C}|| z \mid=1\}$ be a group homomorphism such that $\omega( \pm I)=1$. For any $a=1, \cdots, N$, denote $\omega_{a}=\omega\left(\Omega_{a}\right)$, then $\omega_{a}^{m_{a}}=1$.

For any fixed integer $k$, denote

$$
\mathcal{D}_{k}=\left\{X \in \mathbf{g} \mid \bar{X}=X, \Omega X \Omega^{-1}=\omega(\Omega)^{k} X \text { for any } \Omega \in G_{0}\right\},
$$

then $\left[\mathcal{D}_{j}, \mathcal{D}_{k}\right] \subset \mathcal{D}_{j+k}$. Moreover, if $X \in \mathcal{D}_{k}$, then $X^{2 j-1} \in \mathcal{D}_{(2 j-1) k}$ for any positive integer $j$. Let $\mathcal{D}=\sum_{k=0}^{\infty} \mathcal{D}_{k}$, which is a real Lie subalgebra of $\mathbf{g}$.

Denote $G_{0} \otimes S^{1}=\left\{c g \mid c \in S^{1}, g \in G_{0}\right\}$. For given integer $h$, denote

$$
\begin{gathered}
\Theta_{h}=\left\{\theta \in G_{0} \otimes S^{1} \mid \bar{\theta}=\theta, \widetilde{\omega}(\theta)=1, \theta^{T}=W \theta W^{-1},\right. \\
\text { and } \left.\Omega \theta \Omega^{-1}=\omega(\Omega)^{h} \theta \text { for any } \Omega \in G_{0}\right\} .
\end{gathered}
$$

Here $\widetilde{\omega}: G_{0} \otimes S^{1} \rightarrow S^{1}$ is defined as $\widetilde{\omega}(c g)=\omega(g)$ for any $g \in G_{0}$ and $c \in S^{1}$. It is well-defined since $\omega( \pm I)=1$. Moreover, $\Theta_{h^{\prime}}=\Theta_{h}$ if $h^{\prime} \equiv h \bmod m_{0}$.

$\Theta_{h}$ may be empty. However, $\Theta_{0}$ is always non-empty since $I \in \Theta_{0}$. For $h \neq 0, \Theta_{h}$ is also useful for some nonlinear PDEs. (See the example of the nonlinear Schrödinger equation in Section 6.3.)

Lemma 2 (i) $\theta^{2}= \pm I$ for any $\theta \in \Theta_{h}$.

(ii) $\Theta_{h} \neq \emptyset$ only if $2 h \equiv 0 \bmod m_{0}$.

(iii) $A B=B A$ and $A B \in \Theta_{h}$ hold for any $A \in \Theta_{0}, B \in \Theta_{h}$.

(iv) $\theta X=X \theta$ and $\theta X \in \mathcal{D}_{h+k}$ hold for any $\theta \in \Theta_{h}$ and $X \in \mathcal{D}_{k}$.

Proof: Suppose $\theta=c g$ where $g \in G_{0}$ and $c \in S^{1}$, then by (1) and (5), $W=g^{T} W g=\left(W g W^{-1}\right) W g=W g^{2}$, which implies $g^{2}=I$ and then $\theta^{2}=c^{2} I$. Moreover, $\overline{\theta^{2}}=\theta^{2}$ and $c \in S^{1}$ implies $c^{2}= \pm 1$. Hence (i) is true.

Following (i), (ii) holds since $\omega(\Omega)^{2 h} \theta^{2}=\Omega \theta^{2} \Omega^{-1}=\theta^{2}$ for any $\Omega \in G_{0}$.

Suppose $A \in \Theta_{0}, B \in \Theta_{h}$, then $B A B^{-1}=\widetilde{\omega}(B)^{0} A=A$ implies $A B=B A$. Then it can be checked that $A B \in \Theta_{h}$ by the definition (5). This proves (iii).

Suppose $\theta=c g \in \Theta_{h}$ where $g \in G_{0}$ and $c \in S^{1}$. Since $\omega(g)=1$, we have $\theta X \theta^{-1}=g X g^{-1}=X$, i.e. $\theta X=X \theta$ for any $X \in \mathcal{D}_{k}$. Then $(\theta X)^{T}=X^{T} \theta^{T}=$ $\left(-W X W^{-1}\right)\left(W \theta W^{-1}\right)=-W(\theta X) W^{-1}$. Moreover, $\Omega \theta X \Omega^{-1}=\omega(\Omega)^{h+k} \theta X$ holds for any $\Omega \in G_{0}$. This proves (iv). The lemma is proved. 
Finite dimensional Hamiltonian system related to Lax pair with symmetries

For fixed integers $p$ and $h$, let

$\mathcal{F}_{p, h}=\left\{f(\tau)=\sum_{j=1}^{s} \theta f_{s-j} \tau^{j-1} \mid s\right.$ is a positive integer, $\theta \in \Theta_{h}, f_{s-j} \in \mathbf{R}$,

and $f_{s-j} \neq 0$ holds only when $j$ is even and $\left.h+j \equiv p+1 \bmod m_{0}\right\}$.

Here the necessity of $j$ being even when $f_{s-j} \neq 0$ guarantees that $K^{j-1} \in \mathcal{D}_{j-1}$ when $K \in \mathcal{D}_{1}$.

Note that $\mathcal{F}_{p^{\prime}, h^{\prime}}=\mathcal{F}_{p, h}$ if $p^{\prime} \equiv p \bmod m_{0}$ and $h^{\prime} \equiv h \bmod m_{0}$.

Lemma 3 (i) $p-h$ must be odd if $m_{0}$ is even and $\mathcal{F}_{p, h} \neq\{0\}$.

(ii) $\left[\mathcal{F}_{1,0}, \mathcal{F}_{p, h}\right]=0$ holds for any integers $p$ and $h$.

(iii) If $f \in \mathcal{F}_{p, h}$, then $f(K) \in \mathcal{D}_{p}$ when $K \in \mathcal{D}_{1}$.

Proof: Suppose $f(\tau)=\sum_{j=1}^{s} \theta f_{s-j} \tau^{j-1} \in \mathcal{F}_{p, h}$ and $f \neq 0$. (i) holds since $p-h \equiv j-1 \bmod m_{0}$ and $j-1$ is odd when $f_{s-j} \neq 0$. (ii) follows from (iii) of Lemma 2. Now suppose $f_{s-j} \neq 0$, then $j$ is even and $K^{j-1} \in \mathcal{D}_{j-1}$ since $K \in \mathcal{D}_{1}$. (iv) of Lemma 2 implies $\theta f_{s-j} K^{j-1} \in \mathcal{D}_{h+j-1}=\mathcal{D}_{p}$ by the definition of $\mathcal{F}_{p, h}$. This proves (iii). The lemma is proved.

Lemma 4 Suppose $f \in \mathcal{F}_{1,0}, g \in \mathcal{F}_{p, h}$, then their composition $g \circ f \in \mathcal{F}_{p, h}$.

Proof: Let

$$
f(\tau)=\sum_{j=1}^{s} \theta_{1} f_{s-j} \tau^{j-1}, \quad g(\tau)=\sum_{k=1}^{t} \theta_{2} g_{t-k} \tau^{k-1}
$$

where $\theta_{1} \in \Theta_{0}, \theta_{2} \in \Theta_{h}, f_{s-j} \neq 0$ only if $j$ is even and $j \equiv 2 \bmod m_{0}$, and $g_{t-k} \neq 0$ only if $k$ is even and $k \equiv p+1-h \bmod m_{0}$. Then

$$
\begin{aligned}
& g(f(\tau))=\sum_{k=1}^{t} \theta_{2} g_{t-k}\left(\sum_{j=1}^{s} \theta_{1} f_{s-j} \tau^{j-1}\right)^{k-1} \\
& =\sum_{k=1}^{t} \sum_{j_{1}=1}^{s} \cdots \sum_{j_{k-1}=1}^{s} \theta_{2} \theta_{1}^{k-1} f_{s-j_{1}} \cdots f_{s-j_{k-1}} g_{t-k} \tau^{\left(j_{1}-1\right)+\cdots\left(j_{k-1}-1\right)} .
\end{aligned}
$$

A term in the above summation is nonzero only if $k, j_{1}, \cdots, j_{k-1}$ are all even, $j_{1}, \cdots, j_{k-1} \equiv 2 \bmod m_{0}$ and $k \equiv p+1-h \bmod m_{0}$. Then, $\left(j_{1}-1\right)+\cdots+\left(j_{k-1}-1\right)+1$ is even and $\left(j_{1}-1\right)+\cdots+\left(j_{k-1}-1\right)+1 \equiv p+1-h \bmod m_{0}$. Moreover, (iii) of Lemma 2 implies that $\theta_{2} \theta_{1}^{k-1} \in \Theta_{h}$. Hence $g(f(\tau)) \in \mathcal{F}_{p, h}$.

The space $\mathcal{F}_{p, h}$ will be used in constructing nonlinear constraint in the next section.

In this paper, we will consider the linear system

$$
\Phi_{x}=U(x, \lambda) \Phi
$$

where

$$
U(x, \lambda)=\sum_{j=0}^{p} U_{j}(x) \lambda^{p-j}
$$


with $U_{j} \in \mathcal{D}_{p-j}(j=0,1, \cdots, p)$. Equivalently, $U(x, \lambda)$ satisfies

$$
\begin{aligned}
& \overline{U(\lambda)}=U(\bar{\lambda}), \quad U(\lambda)^{T}=-W U(\lambda) W^{-1}, \\
& \Omega_{a} U(\lambda) \Omega_{a}^{-1}=U\left(\omega_{a} \lambda\right) \quad(a=1, \cdots, N) .
\end{aligned}
$$

Here the first equation in (11) means that the coefficients of $\lambda$ in $U(\lambda)$ are real. The second equation and the third equation mean that $U(\lambda)$ satisfies a symplectic symmetry and cyclic symmetries respectively.

The linear system (9) with symmetries (11) consists of many Lax pairs in $1+1$ dimensions. We will consider this general system in the following Section 3, 4 and 5. The general results to this linear system can be used for some specific integrable systems which will be shown in Section 6 and 7 .

\section{Lax matrix and nonlinear constraint}

Let $\lambda_{1}, \cdots, \lambda_{r}$ be non-zero real numbers such that $\lambda_{j}^{2}$ 's are distinct. For $\sigma=1, \cdots, r$, let $\Phi_{\sigma}=\left(\phi_{1 \sigma}, \cdots, \phi_{2 n, \sigma}\right)^{T}$ be a real column solution of the linear system

$$
\Phi_{\sigma, x}=U\left(x, \lambda_{\sigma}\right) \Phi_{\sigma}
$$

We will construct a finite dimensional Lax matrix first. For given $K \in \mathcal{D}_{1}$, let

$$
L(\lambda)=K+\kappa \sum_{\alpha \in \Sigma_{0}} \sum_{\sigma=1}^{r} \frac{\Omega^{\alpha} \Phi_{\sigma} \Phi_{\sigma}^{T}\left(\Omega^{\alpha}\right)^{T} W}{\lambda-\omega^{\alpha} \lambda_{\sigma}}
$$

where $\kappa$ is a real constant. This construction has already been used in [25, 27] and is similar to those used in constructing Darboux transformations [13, 16, 28].

Lemma $5 L(\lambda)$ satisfies

$$
\begin{aligned}
& L(\bar{\lambda})=\overline{L(\lambda)}, \\
& (L(\lambda))^{T}=-W L(\lambda) W^{-1}, \\
& \Omega_{a} L(\lambda) \Omega_{a}^{-1}=\omega_{a} L\left(\omega_{a} \lambda\right), \quad a=1,2, \cdots, N .
\end{aligned}
$$

Proof: Owing to (3), suppose $\bar{\Omega}_{a}=\varepsilon_{a} \Omega_{a}^{-1}$ with $\varepsilon_{a}= \pm 1$. (14) holds since $\overline{\Omega^{\alpha}}=\varepsilon^{\alpha} \Omega^{-\alpha}, \overline{\omega^{\alpha}}=\omega^{-\alpha}$, and $\varepsilon^{2 \alpha}=1$.

With $W^{T}=-W$, (15) follows from

$$
\begin{aligned}
(L(\lambda))^{T} & =K^{T}-\kappa \sum_{\alpha \in \Sigma_{0}} \sum_{\sigma=1}^{r} \frac{W \Omega^{\alpha} \Phi_{\sigma} \Phi_{\sigma}^{T}\left(\Omega^{\alpha}\right)^{T}}{\lambda-\omega^{\alpha} \lambda_{\sigma}} \\
& =-W K W^{-1}-\kappa W \sum_{\alpha \in \Sigma_{0}} \sum_{\sigma=1}^{r} \frac{\Omega^{\alpha} \Phi_{\sigma} \Phi_{\sigma}^{T}\left(\Omega^{\alpha}\right)^{T} W}{\lambda-\omega^{\alpha} \lambda_{\sigma}} W^{-1} \\
& =-W L(\lambda) W^{-1} .
\end{aligned}
$$


Finite dimensional Hamiltonian system related to Lax pair with symmetries

To prove (16), we have

$$
\begin{aligned}
L\left(\omega_{a} \lambda\right) & =K+\kappa \sum_{\alpha \in \Sigma_{0}} \sum_{\sigma=1}^{r} \frac{\Omega^{\alpha} \Phi_{\sigma} \Phi_{\sigma}^{T}\left(\Omega^{\alpha}\right)^{T} W}{\omega_{a} \lambda-\omega^{\alpha} \lambda_{\sigma}} \\
& =K+\omega_{a}^{-1} \kappa \Omega_{a} \sum_{\alpha \in \Sigma_{0}} \sum_{\sigma=1}^{r} \frac{\Omega^{\alpha} \Phi_{\sigma} \Phi_{\sigma}^{T}\left(\Omega^{\alpha}\right)^{T} \Omega_{a}^{T} W \Omega_{a}}{\lambda-\omega^{\alpha} \lambda_{\sigma}} \Omega_{a}^{-1} \\
& =\omega_{a}^{-1} \Omega_{a} L(\lambda) \Omega_{a}^{-1} .
\end{aligned}
$$

Here we have shifted $\alpha_{a}$ to $\alpha_{a}+1$ at the second equality in (18). The last equality follows from $\Omega_{a} K \Omega_{a}^{-1}=\omega_{a} K$ and $\Omega_{a}^{T} W \Omega_{a}=W$. The lemma is proved.

By Lemma $5, L(\lambda) \in \mathcal{D}$ for any $\lambda \in \mathbf{R}$. Moreover, if $L(\lambda)$ is expanded as $L(\lambda)=\sum_{j=0}^{\infty} \lambda^{-j} L_{j}$ with $L_{0}=K$, then $L_{j} \in \mathcal{D}_{1-j}$.

Corollary 1 Suppose $f \in \mathcal{F}_{p, h}(p \geq 1)$, then $f(L(\lambda))$ satisfies

$$
\begin{aligned}
& f(L(\bar{\lambda}))=\overline{f(L(\lambda))}, \\
& f(L(\lambda))^{T}=-W f(L(\lambda)) W^{-1}, \\
& \Omega_{a} f(L(\lambda)) \Omega_{a}^{-1}=\omega_{a}^{p} f\left(L\left(\omega_{a} \lambda\right)\right), \quad a=1,2, \cdots, N .
\end{aligned}
$$

Proof: Suppose $f(\tau)=\sum_{j=1}^{s} \theta f_{s-j} \tau^{j-1}$ where $\theta \in \Theta_{h}, f_{s-j} \in \mathbf{R}$, then we can check that $f(L(\lambda))=\sum_{j=1}^{s} \theta f_{s-j}(L(\lambda))^{j-1}$ satisfies (19)-(21) by Lemma 5 and the definition of $\Theta_{h}$ and $\mathcal{F}_{p, h}$. This proves the corollary.

For a Laurent series $N(\lambda)=\sum_{j=-\infty}^{n} N_{j} \lambda^{j}$, define

$$
N(\lambda)_{+}=\sum_{j=0}^{n} N_{j} \lambda^{j}, \quad N(\lambda)_{-}=\sum_{j=-\infty}^{-1} N_{j} \lambda^{j}
$$

Write $M(\lambda)=f(L(\lambda))$ and expand it as

$$
M(\lambda)=\sum_{j=0}^{\infty} M_{j} \lambda^{-j}
$$

with $M_{0}=f(K)$. Corollary 1 implies that $M_{j} \in \mathcal{D}_{p-j}$ if $f \in \mathcal{F}_{p, h}$.

Theorem 1 Suppose $f \in \mathcal{F}_{p, h}(p \geq 1)$, then $L(\lambda)$ satisfies

$$
L(\lambda)_{x}=[U(\lambda), L(\lambda)]
$$

under the constraint $U(\lambda)=\widetilde{U}(\lambda)$ where

$$
\widetilde{U}(\lambda)=\left(\lambda^{p} f(L(\lambda))\right)_{+} .
$$

Moreover, $\widetilde{U}(\lambda)=\sum_{j=0}^{p} \widetilde{U}_{j} \lambda^{p-j}$ satisfies $\widetilde{U}_{j} \in \mathcal{D}_{p-j}$. 
Finite dimensional Hamiltonian system related to Lax pair with symmetries

Proof: By using (3), (11) and (13),

$$
\left(\Omega^{\alpha} \Phi_{\sigma} \Phi_{\sigma}^{T}\left(\Omega^{\alpha}\right)^{T} W\right)_{x}=\left[U\left(\omega^{\alpha} \lambda_{\sigma}\right), \Omega^{\alpha} \Phi_{\sigma} \Phi_{\sigma}^{T}\left(\Omega^{\alpha}\right)^{T} W\right] .
$$

Hence

$$
\begin{aligned}
& -L(\lambda)_{x}+[U(\lambda), L(\lambda)]=-\sum_{j=0}^{p}\left[K, \lambda^{p-j} U_{j}\right] \\
& +\kappa \sum_{\alpha \in \Sigma_{0}} \sum_{\sigma=1}^{r} \sum_{j=0}^{p} \frac{\lambda^{p-j}-\left(\omega^{\alpha} \lambda_{\sigma}\right)^{p-j}}{\lambda-\omega^{\alpha} \lambda_{\sigma}}\left[U_{j}, \Omega^{\alpha} \Phi_{\sigma} \Phi_{\sigma}^{T}\left(\Omega^{\alpha}\right)^{T} W\right]
\end{aligned}
$$

is a polynomial of $\lambda$. On the other hand, since $[f(L(\lambda)), L(\lambda)]=0$, we have

$$
\begin{aligned}
& \left(-L(\lambda)_{x}+[\widetilde{U}(\lambda), L(\lambda)]\right)_{+}=\left[\left(\lambda^{p} f(L(\lambda))\right)_{+}, L(\lambda)\right]_{+} \\
& =-\left[\left(\lambda^{p} f(L(\lambda))\right)_{-}, L(\lambda)\right]_{+}=0 .
\end{aligned}
$$

Hence, $L(\lambda)_{x}=[U(\lambda), L(\lambda)]$ holds identically. Moreover, Corollary 1 implies that $\widetilde{U}_{j} \in \mathcal{D}_{p-j}$. The theorem is proved.

Therefore, $L$ satisfies the Lax equation $(24)$ if $U(\lambda)$ satisfies the constraint $U(\lambda)=$ $\left(\lambda^{p} f(L(\lambda))\right)_{+} \cdot$

With the above constraint, (12) becomes a system of nonlinear ODEs

$$
\Phi_{\sigma, x}=\left(\lambda^{p} f\left(L\left(\lambda_{\sigma}\right)\right)\right)_{+} \Phi_{\sigma} .
$$

Theorem 2 Suppose $f \in \mathcal{F}_{p, h}(p \geq 1)$, then (29) is a Hamiltonian system with the Hamiltonian function

$$
H=\frac{1}{2 \kappa m_{1} \cdots m_{N}} \operatorname{tr}\left(\operatorname{Res} \lambda^{p} F(L(\lambda))\right)
$$

where $F$ is a matrix-valued polynomial satisfying $F^{\prime}(\tau)=f(\tau)$ and $F(0)=0$.

Proof: Expand the Lax matrix $L(\lambda)$ as

$$
L(\lambda)=\sum_{j=0}^{\infty} \lambda^{-j} L_{j}
$$

where

$$
L_{0}=K, \quad L_{j}=\kappa \sum_{\alpha \in \Sigma_{0}} \sum_{\sigma=1}^{r}\left(\omega^{\alpha} \lambda_{\sigma}\right)^{j-1} \Omega^{\alpha} \Phi_{\sigma} \Phi_{\sigma}^{T}\left(\Omega^{\alpha}\right)^{T} W \quad(j \geq 1) .
$$

Using the expression $f(\tau)=\sum_{l=1}^{s} \theta f_{s-l} \tau^{l-1}$, we have $F(\tau)=\sum_{l=1}^{s} \frac{1}{l} \theta f_{s-l} \tau^{l}$. 
Finite dimensional Hamiltonian system related to Lax pair with symmetries

Denote $\hat{W}=W^{-1}$, then

$$
\begin{aligned}
& 2 \kappa m_{1} \cdots m_{N} \sum_{k=1}^{2 n} \hat{W}_{j k} \frac{\partial H}{\partial \phi_{k \sigma}} \\
& =\kappa \sum_{\alpha \in \Sigma_{0}} \sum_{a, b, k=1}^{2 n} \sum_{\mu=1}^{+\infty} \operatorname{Res}\left(\lambda^{p-\mu}(f(L(\lambda)))_{a b}\left(\omega^{\alpha} \lambda_{\sigma}\right)^{\mu-1}\right. \\
& \left.\cdot \hat{W}_{j k}\left(\left(\Omega^{\alpha} \Phi_{\sigma}\right)_{b}\left(\left(\Omega^{\alpha}\right)^{T} W\right)_{k a}+\left(\Omega^{\alpha}\right)_{b k}\left(\Phi_{\sigma}^{T}\left(\Omega^{\alpha}\right)^{T} W\right)_{a}\right)\right) \\
& =\kappa \sum_{\alpha \in \Sigma_{0}} \sum_{a, b=1}^{2 n} \sum_{\mu=1}^{+\infty} \operatorname{Res}\left(\lambda^{p-\mu}(f(L(\lambda)))_{a b}\left(\omega^{\alpha} \lambda_{\sigma}\right)^{\mu-1}\right. \\
& \left.\quad \cdot\left(\left(\Omega^{\alpha} \Phi_{\sigma}\right)_{b}\left(\Omega^{-\alpha}\right)_{j a}+\left(\Omega^{-\alpha} W^{-1}\right)_{j b}\left(\Phi_{\sigma}^{T}\left(\Omega^{\alpha}\right)^{T} W\right)_{a}\right)\right) \\
& =2 \kappa \sum_{\alpha \in \Sigma_{0}} \sum_{\mu=1}^{+\infty} \operatorname{Res}\left(\lambda^{p-\mu}\left(\omega^{\alpha} \lambda_{\sigma}\right)^{\mu-1}\left(\Omega^{-\alpha} f(L(\lambda)) \Omega^{\alpha} \Phi_{\sigma}\right)_{j}\right) .
\end{aligned}
$$

Here we have used (3) and (20). Expand

$$
f(L(\lambda))=\sum_{\nu=0}^{\infty} M_{\nu} \lambda^{-\nu}
$$

as in (23), then $M_{j} \in \mathcal{D}_{p-j}$, and

$$
\begin{aligned}
& 2 \kappa m_{1} \cdots m_{N} \sum_{k=1}^{2 n} \hat{W}_{j k} \frac{\partial H}{\partial \phi_{k \sigma}} \\
& =2 \kappa \sum_{\alpha \in \Sigma_{0}} \sum_{\mu=1}^{+\infty} \sum_{\nu=0}^{+\infty} \operatorname{Res}\left(\lambda^{p-\mu-\nu}\left(\omega^{\alpha} \lambda_{\sigma}\right)^{\mu-1}\left(\Omega^{-\alpha} M_{\nu} \Omega^{\alpha} \Phi_{\sigma}\right)_{j}\right) \\
& =2 \kappa \sum_{\alpha \in \Sigma_{0}} \sum_{\nu=0}^{p}\left(\omega^{\alpha} \lambda_{\sigma}\right)^{p-\nu}\left(\Omega^{-\alpha} M_{\nu} \Omega^{\alpha} \Phi_{\sigma}\right)_{j} \\
& =2 \kappa m_{1} \cdots m_{N} \sum_{\nu=0}^{p} \lambda_{\sigma}^{p-\nu}\left(M_{\nu} \Phi_{\sigma}\right)_{j} \\
& =2 \kappa m_{1} \cdots m_{N}\left(\left.\left(\lambda^{p} f(L(\lambda))\right)_{+}\right|_{\lambda=\lambda_{\sigma}} \Phi_{\sigma}\right)_{j} .
\end{aligned}
$$

Therefore, the Hamiltonian equations given by the Hamiltonian function (30) are just (29). The theorem is proved.

In many concrete integrable systems, say, the nonlinear Schrödinger equation in real form, the condition $U_{p-1} \in \mathcal{D}_{p-1} \cap(\text { ker ad } K)^{\perp}$ is needed where $\perp$ refers to the orthogonal compliment with respect to the Killing form $\langle\cdot, \cdot\rangle$ of $\mathbf{g}$. However, usually $M_{1} \in \mathcal{D}_{p-1} \cap(\text { ker ad } K)^{\perp}$ is not guaranteed when $\mathcal{D}_{p-1} \cap$ ker ad $K \neq\{0\}$. This problem can be solved with the help of the following Theorem 3. Before that, we need an algebraic lemma. 
Finite dimensional Hamiltonian system related to Lax pair with symmetries

Lemma 6 Let

$$
\mathcal{M}=\left|\begin{array}{ccccc}
1 & \mu_{1} & \mu_{1}^{2} & \cdots & \mu_{1}^{2 n-1} \\
\vdots & \vdots & \vdots & & \vdots \\
1 & \mu_{n} & \mu_{n}^{2} & \cdots & \mu_{n}^{2 n-1} \\
0 & 1 & 2 \mu_{1} & \cdots & (2 n-1) \mu_{1}^{2 n-2} \\
\vdots & \vdots & \vdots & & \vdots \\
0 & 1 & 2 \mu_{n} & \cdots & (2 n-1) \mu_{n}^{2 n-2}
\end{array}\right|
$$

then

$$
\operatorname{det} \mathcal{M}=(-1)^{n(n-1) / 2} \prod_{1 \leq j<k \leq n}\left(\mu_{k}-\mu_{j}\right)^{4}
$$

Proof: Denote $f(x)=\left(1, x, x^{2}, \cdots, x^{2 n-1}\right)^{T}$,

$\mathcal{M}^{(j, k)}(x)=\operatorname{det}\left(\frac{\mathrm{d}^{j} f}{\mathrm{~d} x^{j}}(x), f\left(\mu_{2}\right), \cdots, f\left(\mu_{n}\right), \frac{\mathrm{d}^{k} f}{\mathrm{~d} x^{k}}(x), \frac{\mathrm{d} f}{\mathrm{~d} x}\left(\mu_{2}\right), \cdots, \frac{\mathrm{d} f}{\mathrm{~d} x}\left(\mu_{n}\right)\right)$.

Then $\mathcal{M}^{(0,1)}\left(\mu_{1}\right)=\mathcal{M}, \mathcal{M}^{(0, k)}\left(\mu_{2}\right)=0, \mathcal{M}^{(1, k)}\left(\mu_{2}\right)=0$, and $\mathcal{M}^{(k, k)}(x)=0$ for any $k \geq 1$. Moreover, we have

$$
\begin{aligned}
& \frac{\mathrm{d}}{\mathrm{d} x} \mathcal{M}^{(0,1)}(x)=\mathcal{M}^{(0,2)}(x), \quad \frac{\mathrm{d}^{2}}{\mathrm{~d} x^{2}} \mathcal{M}^{(0,1)}(x)=\mathcal{M}^{(0,3)}(x)+\mathcal{M}^{(1,2)}(x), \\
& \frac{\mathrm{d}^{3}}{\mathrm{~d} x^{3}} \mathcal{M}^{(0,1)}(x)=\mathcal{M}^{(0,4)}(x)+2 \mathcal{M}^{(1,3)}(x) .
\end{aligned}
$$

This implies $\frac{\mathrm{d}^{k}}{\mathrm{~d} x^{k}} \mathcal{M}^{(0,1)}\left(\mu_{2}\right)=0$ for $k=0,1,2,3$. Since $\mathcal{M}^{(0,1)}(x)$ is a polynomial of $x, \mathcal{M}$ must be of form $\left(\mu_{2}-\mu_{1}\right)^{4} F_{1}\left(\mu_{1}, \cdots, \mu_{n}\right)$ where $F_{1}$ is a polynomial. Owing to the symmetry, $\mathcal{M}=\prod_{1 \leq j<k \leq n}\left(\mu_{k}-\mu_{j}\right)^{4} F_{2}\left(\mu_{1}, \cdots, \mu_{n}\right)$ where $F_{2}$ is another polynomial. However, regarded as a polynomial of $\mu_{1}, \mathcal{M}$ is of degree $4 n-4$. Hence $F_{2}$ must be a constant. Comparing the coefficient of $\prod_{k=2}^{n} \mu_{k}^{2 k-4}$, we get $F_{2}=(-1)^{n(n-1) / 2}$. The lemma is proved.

Theorem 3 Suppose $K \in \mathcal{D}_{1}$ is diagonalizable, $f \in \mathcal{F}_{p, h}(p \geq 1)$. Expand $M(\lambda)=$ $f(L(\lambda))$ as in (23) where $L(\lambda)$ is given by (13). Then there exists a polynomial $\zeta$ such that $\widetilde{M}(\lambda) \equiv \zeta(M(\lambda))=M_{0}+\lambda^{-1} \widetilde{M}_{1}+o\left(\lambda^{-1}\right)$ with $\widetilde{M}_{1} \in \mathcal{D}_{p-1} \cap(\operatorname{ker} \text { ad } K)^{\perp}$ and $M_{1}-\widetilde{M}_{1} \in \mathcal{D}_{p-1} \cap \operatorname{ker} \operatorname{ad} K$.

Proof: Let $K=T \Lambda T^{-1}$ where $\Lambda$ is a complex diagonal matrix and $T$ is a complex invertible matrix. Let $\widetilde{m}_{0}=m_{0}$ if $m_{0}$ is even and $\widetilde{m}_{0}=2 m_{0}$ if $m_{0}$ is odd. Let $\mu_{1}, \cdots, \mu_{l}$ be all the distinct eigenvalues of $\Lambda^{\widetilde{m}_{0}}$. By Lemma 6 , there is a unique complex solution $\zeta_{j}(j=0,1, \cdots, 2 l-1)$ of the linear system

$$
\sum_{k=0}^{2 l-1} \zeta_{k} \mu_{j}^{k}=0, \quad \widetilde{m}_{0} \mu_{j} \sum_{k=0}^{2 l-1} k \zeta_{k} \mu_{j}^{k-1}=1 \quad(j=1, \cdots, l)
$$


Finite dimensional Hamiltonian system related to Lax pair with symmetries

Then

$$
\sum_{k=0}^{2 l-1} \zeta_{k} K^{k \widetilde{m}_{0}}=0, \quad \widetilde{m}_{0} \sum_{k=0}^{2 l-1} k \zeta_{k} K^{k \widetilde{m}_{0}}=I
$$

Since $K$ is real and $\zeta_{j}$ 's are unique, $\zeta_{j}$ 's must be real.

Let $\zeta(\tau)=\tau-\sum_{k=0}^{2 l-1} \zeta_{k} \tau^{k \widetilde{m}_{0}+1}$, then $\zeta \in \mathcal{F}_{1,0}$ since $\widetilde{m}_{0}$ is always even, and $\zeta(K)=K$.

For any $H \in \operatorname{ker} \operatorname{ad} K$,

$$
\begin{aligned}
& \langle H, \widetilde{M}-M\rangle=-\sum_{k=0}^{2 l-1} \zeta_{k}\left\langle H, M^{k \widetilde{m}_{0}+1}\right\rangle \\
& =-\sum_{k=0}^{2 l-1} \zeta_{k}\left\langle H, K^{k \widetilde{m}_{0}+1}+\lambda^{-1} \sum_{j=0}^{k \widetilde{m}_{0}} K^{j} M_{1} K^{k \widetilde{m}_{0}-j}\right\rangle+o\left(\lambda^{-1}\right) \\
& =-\sum_{k=0}^{2 l-1}\left\langle H, \zeta_{k} K^{k \widetilde{m}_{0}+1}+\lambda^{-1}\left(k \widetilde{m}_{0}+1\right) \zeta_{k} K^{k \widetilde{m}_{0}} M_{1}\right\rangle+o\left(\lambda^{-1}\right) \\
& =-\lambda^{-1}\left\langle H, M_{1}\right\rangle+o\left(\lambda^{-1}\right) .
\end{aligned}
$$

Comparing the coefficients of $\lambda^{-1}$, we have $\left\langle H, \widetilde{M}_{1}\right\rangle=0$. Since $\zeta \in \mathcal{F}_{1,0}$, Lemma 4 and Corollary 1 imply that $\widetilde{M}_{1} \in \mathcal{D}_{p-1}$. Hence $\widetilde{M}_{1} \in \mathcal{D}_{p-1} \cap(\operatorname{ker} \operatorname{ad} K)^{\perp}$.

On the other hand,

$$
\begin{aligned}
& {[K, \widetilde{M}-M]=-\sum_{k=0}^{2 l-1} \zeta_{k}\left[K, K^{k \widetilde{m}_{0}+1}+\lambda^{-1} \sum_{j=0}^{k \widetilde{m}_{0}} K^{j} M_{1} K^{k \widetilde{m}_{0}-j}\right]+o\left(\lambda^{-1}\right)} \\
& =-\lambda^{-1} \sum_{k=0}^{2 l-1}\left[\zeta_{k} K^{k \widetilde{m}_{0}+1}, M_{1}\right]+o\left(\lambda^{-1}\right)=o\left(\lambda^{-1}\right) .
\end{aligned}
$$

Comparing the coefficients of $\lambda^{-1}$, we get $M_{1}-\widetilde{M}_{1} \in \mathcal{D}_{p-1} \cap$ ker ad $K$. The theorem is proved.

Owing to Lemma 4 and Theorem 3, we can always want $U_{1} \in \mathcal{D}_{p-1} \cap(\operatorname{ker} \operatorname{ad} K)^{\perp}$ if necessary, by replacing $f$ with $f \circ \zeta$. However, when $\zeta$ is complicated, the Hamiltonian function need not be calculated from Theorem 2. Instead, it is simpler to integrate it from the Hamiltonian equations directly. In this case, Theorem 2 is still important because it shows that the Hamiltonian function is expressed by the Lax matrix, which is essential in the proof of Liouville integrability.

\section{4. $r$ matrix}

In $\mathbf{R}^{2 n \times r}$ with coordinates $\phi_{j \sigma}(j=1, \cdots, 2 n ; \sigma=1, \cdots, r)$, define the symplectic form

$$
\sum_{j, k=1}^{2 n} \sum_{\sigma=1}^{r} W_{j k} \mathrm{~d} \phi_{j \sigma} \wedge \mathrm{d} \phi_{k \sigma}
$$


Finite dimensional Hamiltonian system related to Lax pair with symmetries

Then, for any two smooth functions $f$ and $g$, their Poisson bracket is

$$
\{f, g\}=\sum_{j, k=1}^{2 n} \sum_{\sigma=1}^{r} \hat{W}_{j k} \frac{\partial f}{\partial \phi_{j \sigma}} \frac{\partial g}{\partial \phi_{k \sigma}}
$$

with $\hat{W}=W^{-1}$.

Theorem 4 For any $\lambda, \mu \in \mathbf{C}$,

$\left\{L_{a b}(\lambda), L_{c d}(\mu)\right\}=\left[r_{1}(\lambda, \mu), L(\lambda) \otimes I\right]_{a b c d}+\left[r_{2}(\lambda, \mu), I \otimes L(\mu)\right]_{a b c d}$

holds where the Poisson bracket is given by (45) and

$$
\begin{aligned}
&\left(r_{1}(\lambda, \mu)\right)_{a b c d}=\sum_{\gamma \in \Sigma_{0}} \frac{\kappa}{\mu-\omega^{\gamma} \lambda}\left(\left(\Omega^{-\gamma}\right)_{a d}\left(\Omega^{\gamma}\right)_{c b}-\left(\Omega^{-\gamma} W^{-1}\right)_{a c}\left(W \Omega^{\gamma}\right)_{d b}\right) \\
&\left(r_{2}(\lambda, \mu)\right)_{a b c d}=\sum_{\gamma \in \Sigma_{0}} \frac{\kappa \omega^{\gamma}}{\mu-\omega^{\gamma} \lambda}\left(\left(\Omega^{-\gamma}\right)_{a d}\left(\Omega^{\gamma}\right)_{c b}-\left(\Omega^{-\gamma} W^{-1}\right)_{a c}\left(W \Omega^{\gamma}\right)_{d b}\right) \\
&=-\left(r_{1}(\mu, \lambda)\right)_{c d a b} .
\end{aligned}
$$

Here

$$
[A, B]_{a b c d}=\sum_{p, q=1}^{2 n}\left(A_{a p c q} B_{p b q d}-B_{a p c q} A_{p b q d}\right)
$$

for any two $(2 n)^{2} \times(2 n)^{2}$ matrices $A$ and $B$.

Proof: Written in components,

$$
\begin{aligned}
& L_{a b}(\lambda)=K_{a b}+\sum_{\alpha \in \Sigma_{0}} \sum_{\sigma=1}^{r} \sum_{f, g, h=1}^{2 n} \frac{\kappa\left(\Omega^{\alpha}\right)_{a f} \phi_{f \sigma} \phi_{g \sigma}\left(\Omega^{\alpha}\right)_{h g} W_{h b}}{\lambda-\omega^{\alpha} \lambda_{\sigma}}, \\
& \frac{\partial L_{a b}(\lambda)}{\partial \phi_{j \sigma}}=\sum_{\alpha \in \Sigma_{0}} \sum_{f, h=1}^{2 n}\left(\frac{\kappa\left(\Omega^{\alpha}\right)_{a j} \phi_{f \sigma}\left(\Omega^{\alpha}\right)_{h f} W_{h b}}{\lambda-\omega^{\alpha} \lambda_{\sigma}}+\frac{\kappa\left(\Omega^{\alpha}\right)_{a f} \phi_{f \sigma}\left(\Omega^{\alpha}\right)_{h j} W_{h b}}{\lambda-\omega^{\alpha} \lambda_{\sigma}}\right), \\
& \frac{\partial L_{c d}(\mu)}{\partial \phi_{k \sigma}}=\sum_{\beta \in \Sigma_{0}} \sum_{q, r=1}^{2 n}\left(\frac{\kappa\left(\Omega^{\beta}\right)_{c k} \phi_{q \sigma}\left(\Omega^{\beta}\right)_{r q} W_{r d}}{\mu-\omega^{\beta} \lambda_{\sigma}}+\frac{\kappa\left(\Omega^{\beta}\right)_{c q} \phi_{q \sigma}\left(\Omega^{\beta}\right)_{r k} W_{r d}}{\mu-\omega^{\beta} \lambda_{\sigma}}\right) .
\end{aligned}
$$

The Poisson bracket is

$$
\begin{aligned}
\Delta_{a b c d} \equiv & \left\{L_{a b}(\lambda), L_{c d}(\mu)\right\}=\sum_{\sigma=1}^{r} \sum_{j, k=1}^{2 n} \hat{W}_{j k} \frac{\partial L_{a b}(\lambda)}{\partial \phi_{j \sigma}} \frac{\partial L_{c d}(\mu)}{\partial \phi_{k \sigma}} \\
= & \sum_{\sigma=1}^{r} \sum_{\alpha, \beta \in \Sigma_{0}} \sum_{j, k, f, h, q, r=1}^{2 n} \kappa^{2}\left(\frac{\omega^{\alpha}}{\lambda-\omega^{\alpha} \lambda_{\sigma}}-\frac{\omega^{\beta}}{\mu-\omega^{\beta} \lambda_{\sigma}}\right) \frac{\phi_{f \sigma} \phi_{q \sigma}}{\omega^{\alpha} \mu-\omega^{\beta} \lambda} \hat{W}_{j k} \\
& \cdot\left(\left(\Omega^{\alpha}\right)_{a j}\left(\Omega^{\alpha}\right)_{h f} W_{h b}+\left(\Omega^{\alpha}\right)_{a f}\left(\Omega^{\alpha}\right)_{h j} W_{h b}\right) \\
& \cdot\left(\left(\Omega^{\beta}\right)_{c k}\left(\Omega^{\beta}\right)_{r q} W_{r d}+\left(\Omega^{\beta}\right)_{c q}\left(\Omega^{\beta}\right)_{r k} W_{r d}\right) \\
= & \kappa^{2} \sum_{\sigma=1}^{r} \sum_{\alpha, \beta \in \Sigma_{0}}\left(\frac{1}{\mu-\omega^{\beta-\alpha} \lambda} \frac{1}{\lambda-\omega^{\alpha} \lambda_{\sigma}}+\frac{1}{\lambda-\omega^{\alpha-\beta} \mu} \frac{1}{\mu-\omega^{\beta} \lambda_{\sigma}}\right) D_{a b c d \alpha \beta}
\end{aligned}
$$


Finite dimensional Hamiltonian system related to Lax pair with symmetries

where

$$
\begin{gathered}
D_{a b c d \alpha \beta}=\left(\Omega^{\alpha-\beta} W^{-1}\right)_{a c}\left(W^{T} \Omega^{\alpha} \Phi_{\sigma} \Phi_{\sigma}^{T}\left(\Omega^{\beta}\right)^{T} W\right)_{b d}+\left(\Omega^{\alpha-\beta}\right)_{a d}\left(W^{T} \Omega^{\alpha} \Phi_{\sigma} \Phi_{\sigma}^{T}\left(\Omega^{\beta}\right)^{T}\right)_{b c} \\
-\left(\Omega^{\beta-\alpha}\right)_{c b}\left(\Omega^{\alpha} \Phi_{\sigma} \Phi_{\sigma}^{T}\left(\Omega^{\beta}\right)^{T} W\right)_{a d}-\left(W \Omega^{\alpha-\beta}\right)_{b d}\left(\Omega^{\alpha} \Phi_{\sigma} \Phi_{\sigma}^{T}\left(\Omega^{\beta}\right)^{T}\right)_{a c} .
\end{gathered}
$$

Here we have used (3).

Let $\gamma=\beta-\alpha, \Pi_{\alpha}^{(\sigma)}=\Omega^{\alpha} \Phi_{\sigma} \Phi_{\sigma}^{T}\left(\Omega^{\alpha}\right)^{T} W$, then $\left(\Pi_{\alpha}^{(\sigma)}\right)^{T}=-W \Pi_{\alpha}^{(\sigma)} W^{-1}, \Pi_{\beta}^{(\sigma)}=$ $\Omega^{\gamma} \prod_{\alpha}^{(\sigma)} \Omega^{-\gamma}$.

Written in terms of $\Pi_{\alpha}^{(\sigma)}$,

$$
\begin{aligned}
D_{a b c d \alpha \beta}= & -\left(\Omega^{-\gamma} W^{-1}\right)_{a c}\left(W \Omega^{\gamma} \Pi_{\alpha}^{(\sigma)}\right)_{d b}+\left(\Omega^{-\gamma}\right)_{a d}\left(\Omega^{\gamma} \Pi_{\alpha}^{(\sigma)}\right)_{c b} \\
& -\left(\Omega^{\gamma}\right)_{c b}\left(\Pi_{\alpha}^{(\sigma)} \Omega^{-\gamma}\right)_{a d}+\left(W \Omega^{\gamma}\right)_{d b}\left(\Pi_{\alpha}^{(\sigma)} \Omega^{-\gamma} W^{-1}\right)_{a c} .
\end{aligned}
$$

On the other hand, written in terms of $\Pi_{\beta}^{(\sigma)}$,

$$
\begin{aligned}
D_{a b c d \alpha \beta}= & -\left(\Omega^{-\gamma} W^{-1}\right)_{a c}\left(W \Omega^{-\gamma} \Pi_{\beta}^{(\sigma)}\right)_{b d}+\left(\Omega^{-\gamma}\right)_{a d}\left(\Pi_{\beta}^{(\sigma)} \Omega^{\gamma}\right)_{c b} \\
& -\left(\Omega^{\gamma}\right)_{c b}\left(\Omega^{-\gamma} \Pi_{\beta}^{(\sigma)}\right)_{a d}+\left(W \Omega^{\gamma}\right)_{d b}\left(\Pi_{\beta}^{(\sigma)} \Omega^{\gamma} W^{-1}\right)_{c a} .
\end{aligned}
$$

Hence

$$
\begin{aligned}
\Delta_{a b c d}= & \sum_{\gamma \in \Sigma_{0}} \sum_{l=1}^{2 n} \frac{\kappa}{\mu-\omega^{\gamma} \lambda} \\
& \cdot\left(-\left(\Omega^{-\gamma} W^{-1}\right)_{a c}\left(W \Omega^{\gamma}(L(\lambda)-K)\right)_{d b}+\left(\Omega^{-\gamma}\right)_{a d}\left(\Omega^{\gamma}(L(\lambda)-K)\right)_{c b}\right. \\
& \left.-\left(\Omega^{\gamma}\right)_{c b}\left((L(\lambda)-K) \Omega^{-\gamma}\right)_{a d}+\left(W \Omega^{\gamma}\right)_{d b}\left((L(\lambda)-K) \Omega^{-\gamma} W^{-1}\right)_{a c}\right) \\
& +\sum_{\gamma \in \Sigma_{0}} \sum_{l=1}^{2 n} \frac{\kappa \omega^{\gamma}}{\mu-\omega^{\gamma} \lambda} \\
& \cdot\left(-\left(\Omega^{-\gamma} W^{-1}\right)_{a c}\left(W \Omega^{-\gamma}(L(\mu)-K)\right)_{b d}+\left(\Omega^{-\gamma}\right)_{a d}\left((L(\mu)-K) \Omega^{\gamma}\right)_{c b}\right. \\
& \left.-\left(\Omega^{\gamma}\right)_{c b}\left(\Omega^{-\gamma}(L(\mu)-K)\right)_{a d}+\left(W \Omega^{\gamma}\right)_{d b}\left((L(\mu)-K) \Omega^{\gamma} W^{-1}\right)_{c a}\right) .
\end{aligned}
$$

In $\Delta_{a b c d}$, the terms with $K_{j k}$ 's are

$$
\begin{aligned}
& \sum_{\gamma \in \Sigma_{0}} \frac{\kappa}{\mu-\omega^{\gamma} \lambda}\left(\left(\Omega^{-\gamma} W^{-1}\right)_{a c}\left(W \Omega^{\gamma} K\right)_{d b}-\left(\Omega^{-\gamma}\right)_{a d}\left(\Omega^{\gamma} K\right)_{c b}\right. \\
& \left.+\left(\Omega^{\gamma}\right)_{c b}\left(K \Omega^{-\gamma}\right)_{a d}-\left(W \Omega^{\gamma}\right)_{d b}\left(K \Omega^{-\gamma} W^{-1}\right)_{a c}\right) \\
& +\sum_{\gamma \in \Sigma_{0}} \frac{\kappa \omega^{\gamma}}{\mu-\omega^{\gamma} \lambda}\left(\left(\Omega^{-\gamma} W^{-1}\right)_{a c}\left(W \Omega^{-\gamma} K\right)_{b d}-\left(\Omega^{-\gamma}\right)_{a d}\left(K \Omega^{\gamma}\right)_{c b}\right. \\
& \left.+\left(\Omega^{\gamma}\right)_{c b}\left(\Omega^{-\gamma} K\right)_{a d}-\left(W \Omega^{\gamma}\right)_{d b}\left(K \Omega^{\gamma} W^{-1}\right)_{a c}\right)=0,
\end{aligned}
$$

in which we have used the relations in (3) and the fact $K \in \mathcal{D}_{1}$. Hence

$$
\begin{aligned}
\Delta_{a b c d}= & \sum_{\gamma \in \Sigma_{0}} \sum_{l=1}^{2 n} \frac{\kappa}{\mu-\omega^{\gamma} \lambda}\left(-\left(\Omega^{-\gamma} W^{-1}\right)_{a c}\left(W \Omega^{\gamma}\right)_{d l} L_{l b}(\lambda)+\left(\Omega^{-\gamma}\right)_{a d}\left(\Omega^{\gamma}\right)_{c l} L_{l b}(\lambda)\right. \\
& \left.-L_{a l}(\lambda)\left(\Omega^{-\gamma}\right)_{l d}\left(\Omega^{\gamma}\right)_{c b}+L_{a l}(\lambda)\left(\Omega^{-\gamma} W^{-1}\right)_{l c}\left(W \Omega^{\gamma}\right)_{d b}\right) \\
+ & \sum_{\gamma \in \Sigma_{0}} \sum_{l=1}^{2 n} \frac{\kappa \omega^{\gamma}}{\mu-\omega^{\gamma} \lambda}\left(-\left(\Omega^{-\gamma} W^{-1}\right)_{a c}\left(W \Omega^{\gamma}\right)_{l b} L_{l d}(\mu)+\left(\Omega^{-\gamma}\right)_{a l}\left(\Omega^{\gamma}\right)_{c b} L_{l d}(\mu)\right. \\
& \left.-L_{c l}(\mu)\left(\Omega^{-\gamma}\right)_{a d}\left(\Omega^{\gamma}\right)_{l b}+L_{c l}(\mu)\left(\Omega^{-\gamma} W^{-1}\right)_{a l}\left(W \Omega^{\gamma}\right)_{d b}\right)
\end{aligned}
$$


Finite dimensional Hamiltonian system related to Lax pair with symmetries

which is the result of the theorem.

From Theorem 4, it is easy to derive

Theorem 5 Suppose $\theta_{1}$ and $\theta_{2}$ are constant $2 n \times 2 n$ matrices such that $\left[\theta_{j}, L(\lambda)\right]=0$ $(j=1,2)$, then $\left\{\operatorname{tr}\left(\theta_{1} L(\lambda)^{k}\right), \operatorname{tr}\left(\theta_{2} L(\mu)^{l}\right)\right\}=0$ holds for any positive integers $k$ and $l$, and complex numbers $\lambda$ and $\mu$.

Proof: According to Theorem 4,

$$
\begin{aligned}
& \frac{1}{k l}\left\{\operatorname{tr}\left(\theta_{1} L(\lambda)^{k}\right), \operatorname{tr}\left(\theta_{2} \theta L(\mu)^{l}\right)\right\} \\
= & \sum_{a, b, c, d=1}^{2 n}\left(\theta_{1} L(\lambda)^{k-1}\right)_{b a}\left(\theta_{2} L(\mu)^{l-1}\right)_{d c}\left\{L(\lambda)_{a b}, L(\mu)_{c d}\right\} \\
= & \sum_{a, b, c, d, j=1}^{2 n}\left(\theta_{1} L(\lambda)^{k-1}\right)_{b a}\left(\theta_{2} L(\mu)^{l-1}\right)_{d c} \\
& \cdot\left(\left(r_{1}\right)_{a j c d} L(\lambda)_{j b}-L(\lambda)_{a j}\left(r_{1}\right)_{j b c d}+\left(r_{2}\right)_{a b c j} L(\mu)_{j d}-L(\mu)_{c j}\left(r_{2}\right)_{a b j d}\right) \\
= & 0
\end{aligned}
$$

by the relations

$$
\sum_{b=1}^{2 n}\left(\theta_{1} L(\lambda)^{k-1}\right)_{b a} L(\lambda)_{j b}=\left(\theta_{1} L(\lambda)^{k}\right)_{j a}
$$

etc. The theorem is proved.

According to Theorem 2 and $5,\left\{H, \operatorname{tr}\left(\theta L(\lambda)^{k}\right)\right\}=0$ holds for any positive integer $k$, complex number $\lambda$ and matrix $\theta$ with $[\theta, L(\lambda)]=0$.

\section{Independence of conserved integrals}

According to $(15), \operatorname{tr}\left(\theta L(\lambda)^{2 k-1}\right)=0$ for any $K \in \mathcal{D}_{1}, \theta \in \Theta_{h}$, and positive integers $k$ and $h$. It is only necessary to consider $\operatorname{tr}\left(\theta L(\lambda)^{k}\right)$ for even $k$ to generate the conserved integrals.

For given $\theta \in \Theta_{h}$, expand

$$
\operatorname{tr}\left(\theta L(\lambda)^{2 k}\right)=\sum_{j=0}^{\infty} s_{j}^{(2 k)}(\theta) \lambda^{-j} .
$$

By $(16), s_{j}^{(2 k)}(\theta)=\omega_{a}^{2 k+h-j} s_{j}^{(2 k)}(\theta)$ for all $a=1, \cdots, N$. Hence $s_{j}^{(2 k)}(\theta)=0$ unless $j \equiv 2 k+h \bmod m_{0}$.

To consider the non-zero $s_{j}^{(2 k)}(\theta)$ 's, let

$$
E_{p}^{(k)}(\theta)=\frac{1}{2 k} s_{m_{0}(p-1)+2 k+h}^{(2 k)}(\theta) .
$$

Theorem 6 Suppose $K \in \mathcal{D}_{1}$ is diagonalizable. Suppose also that there exist $\theta_{k} \in \Theta_{h_{k}}$ $(k=1, \cdots, n)$ such that $\left[\theta_{j}, \theta_{k}\right]=0$ for all $j, k$, and $\theta_{j} K^{2 j-1}(j=1, \cdots, n)$ are linearly independent. Then $E_{p}^{(k)}\left(\theta_{k}\right)(k=1, \cdots, n ; p=1, \cdots, r)$ are functionally independent in a dense open subset of $\mathbf{R}^{2 n \times r}$. 
Finite dimensional Hamiltonian system related to Lax pair with symmetries

Proof: According to (iv) of Lemma 2, $\left[\theta_{j}, K\right]=0$ for all $j$ since $\theta_{j} \in \Theta_{h_{j}}$ and $K \in \mathcal{D}_{1}$. Moreover, $\theta_{j}^{2}= \pm I$ implies that $\theta_{j}$ 's are diagonalizable. Hence there exists a $2 n \times 2 n$ complex invertible matrix $T$ such that $K^{(0)}=T K T^{-1}$ and $\theta_{j}^{(0)}=T \theta_{j} T^{-1}$ are all complex diagonal matrices. Let $\Xi=\left(\xi_{j k}\right)_{1 \leq j \leq n, 1 \leq k \leq 2 n}$ where $\xi_{j k}$ is the $(k, k)$ entry of $\theta_{j}^{(0)}\left(K^{(0)}\right)^{2 j-1}$. Since $\theta_{j} K^{2 j-1}(j=1, \cdots, n)$ are linearly independent, $\operatorname{rank}(\Xi)=n$. Without loss of generality, suppose that the first $n$ columns of $\Xi$ are linearly independent.

Let

$$
S=\left\{\Psi \in \mathbf{R}^{2 n \times r} \mid \text { all the entries of } T \Psi \text { are non-zero }\right\},
$$

then $S$ is a dense subset of $\mathbf{R}^{2 n \times r}$.

Now we compute the Jacobian matrix of $E_{p}^{(k)}\left(\theta_{k}\right)$ with respect to $\phi_{j \sigma}$. By the definition of $L(\lambda)$,

$$
\begin{aligned}
& \frac{1}{2 k} \operatorname{tr}\left(\theta_{k} L(\lambda)^{2 k}\right)=\frac{1}{2 k} \operatorname{tr}\left(\theta_{k}\left(K+\kappa \sum_{\alpha \in \Sigma_{0}} \sum_{\sigma=1}^{r} \frac{\Omega^{\alpha} \Phi_{\sigma} \Phi_{\sigma}^{T}\left(\Omega^{\alpha}\right)^{T} W}{\lambda-\omega^{\alpha} \lambda_{\sigma}}\right)^{2 k}\right) \\
& =\frac{1}{2 k} \operatorname{tr}\left(\theta_{k} K^{2 k}+2 k \kappa \theta_{k} K^{2 k-1} \sum_{\alpha \in \Sigma_{0}} \sum_{\sigma=1}^{r} \frac{\Omega^{\alpha} \Phi_{\sigma} \Phi_{\sigma}^{T}\left(\Omega^{\alpha}\right)^{T} W}{\lambda-\omega^{\alpha} \lambda_{\sigma}}\right)+\cdots
\end{aligned}
$$

where "..." represents the terms of $\phi_{j \sigma}$ 's whose degrees are higher than 2 . Hence

$$
\begin{aligned}
& E_{p}^{(k)}\left(\theta_{k}\right)=\kappa \operatorname{tr}\left(\sum_{\alpha \in \Sigma_{0}} \sum_{\sigma=1}^{r}\left(\omega^{\alpha} \lambda_{\sigma}\right)^{m_{0}(p-1)+2 k+h_{k}-1} \theta_{k} K^{2 k-1} \Omega^{\alpha} \Phi_{\sigma} \Phi_{\sigma}^{T}\left(\Omega^{\alpha}\right)^{T} W\right)+\cdots \\
& =\kappa m_{1} \cdots m_{N} \sum_{\sigma=1}^{r} \lambda_{\sigma}^{m_{0}(p-1)+2 k+h_{k}-1} \Phi_{\sigma}^{T} W \theta_{k} K^{2 k-1} \Phi_{\sigma}+\cdots
\end{aligned}
$$

since $\omega^{m_{0} \alpha}=1, K \in \mathcal{D}_{1}, \theta_{k} \in \Theta_{h_{k}}$ and (3) holds.

Denote $\Psi=\left(\phi_{j \sigma}\right)_{1 \leq j \leq 2 n ; 1 \leq \sigma \leq r}$. For $k=1, \cdots, n, j=1, \cdots, 2 n, p=1, \cdots, r$, $\sigma=1, \cdots, r$,

$$
\frac{\partial E_{p}^{(k)}\left(\theta_{k}\right)}{\partial \phi_{j \sigma}}=2 \kappa m_{1} \cdots m_{N} \lambda_{\sigma}^{m_{0}(p-1)+2 k+h_{k}-1}\left(W \theta_{k} K^{2 k-1} \Psi\right)_{j \sigma}+\cdots
$$

Here we have used the fact that $W \theta_{k} K^{2 k-1}$ is symmetric. Then

$$
\sum_{l=1}^{2 n}\left(W^{-1}\right)_{j l} \frac{\partial E_{p}^{(k)}\left(\theta_{k}\right)}{\partial \phi_{l \sigma}}=2 \kappa m_{1} \cdots m_{N} \lambda_{\sigma}^{m_{0}(p-1)+2 k+h_{k}-1}\left(\theta_{k} K^{2 k-1} \Psi\right)_{j \sigma}+\cdots .
$$

Let

$$
\mathcal{M}^{(s)}=\left(\lambda_{\sigma}^{m_{0}(p-1)+2 k+h_{k}-1}\left(\theta_{k} K^{2 k-1} \Psi\right)_{j \sigma}\right)_{n s \times 2 n s} \quad(1 \leq s \leq r)
$$

where the row indices are $k=1, \cdots, n$ and $p=1, \cdots, s$, and the column indices are $j=$ $1, \cdots, 2 n$ and $\sigma=1, \cdots, s$. Write $\mathcal{M}^{(s)}$ as the block matrix $\mathcal{M}^{(s)}=\left(\mathcal{M}_{k j}^{(s)}\right)_{1 \leq k \leq n ; 1 \leq j \leq 2 n}$ where

$$
\mathcal{M}_{k j}^{(s)}=\left(\begin{array}{ccc}
\lambda_{1}^{2 k+h_{k}-1}\left(\Psi^{(k)}\right)_{j 1} & \cdots & \lambda_{s}^{2 k+h_{k}-1}\left(\Psi^{(k)}\right)_{j s} \\
\lambda_{1}^{m_{0}+2 k+h_{k}-1}\left(\Psi^{(k)}\right)_{j 1} & \cdots & \lambda_{s}^{m_{0}+2 k+h_{k}-1}\left(\Psi^{(k)}\right)_{j s} \\
\vdots & & \vdots \\
\lambda_{1}^{m_{0}(s-1)+2 k+h_{k}-1}\left(\Psi^{(k)}\right)_{j 1} & \cdots & \lambda_{s}^{m_{0}(s-1)+2 k+h_{k}-1}\left(\Psi^{(k)}\right)_{j s}
\end{array}\right)
$$


Finite dimensional Hamiltonian system related to Lax pair with symmetries are $s \times s$ matrices, and $\Psi^{(k)}=\theta_{k} K^{2 k-1} \Psi$. Let

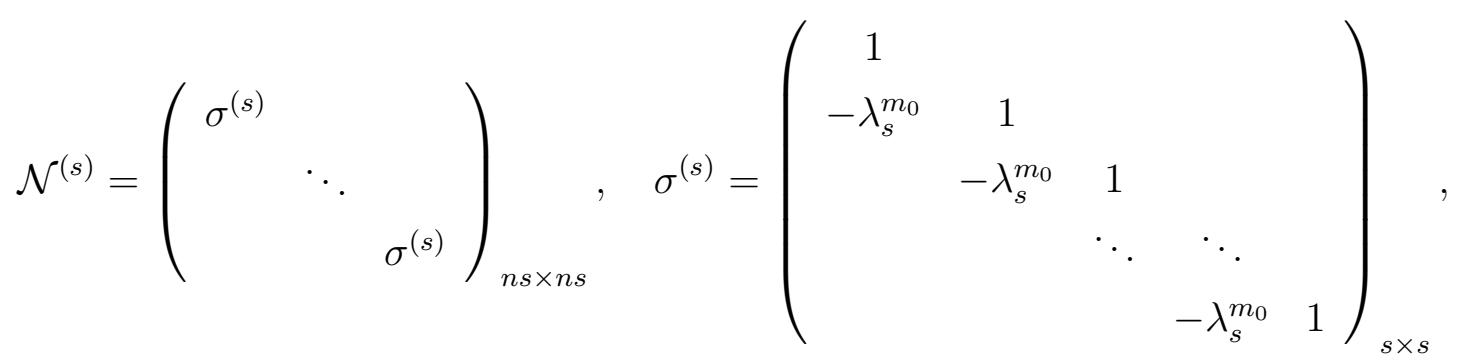

then

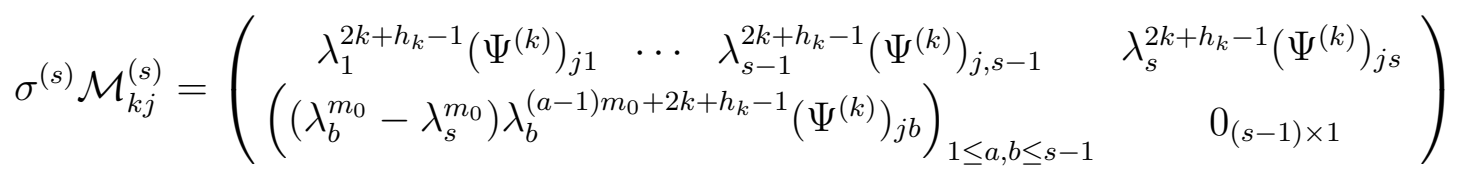

and $\mathcal{M}^{(s)}$ is transformed to $\mathcal{N}^{(s)} \mathcal{M}^{(s)}$ under elementary transformations. Take another elementary transformation for $\mathcal{N}^{(s)} \mathcal{M}^{(s)}$ by changing the 1st, $(s+1)$-th, $(2 s+1)$-th, $\cdots$, $((n-1) s+1)$-th rows to the bottom and changing the $s$-th, $2 s$-th, $\cdots, 2 n s$-th column to the right. Then $\mathcal{M}^{(s)}$ is changed to

$$
\left(\begin{array}{cc}
\widetilde{M}^{(s-1)} & 0 \\
* & B_{s}
\end{array}\right)
$$

where $\widetilde{\mathcal{M}}^{(s-1)}=\left(\widetilde{M}_{k j}^{(s-1)}\right)_{1 \leq k \leq n, 1 \leq j \leq 2 n}$,

$$
\begin{aligned}
& \widetilde{\mathcal{M}}_{k j}^{(s-1)}=\mathcal{M}_{k j}^{(s-1)}\left(\begin{array}{lll}
\lambda_{1}^{m_{0}}-\lambda_{s}^{m_{0}} & & \\
& \ddots & \\
& & \lambda_{s-1}^{m_{0}}-\lambda_{s}^{m_{0}}
\end{array}\right), \\
& B_{s}=\left(\lambda_{s}^{2 k+h_{k}-1}\left(\Psi^{(k)}\right)_{j s}\right)_{1 \leq k \leq n, 1 \leq j \leq 2 n}
\end{aligned}
$$

Then

$\sum_{l=1}^{2 n} T_{j l}\left(B_{s}\right)_{k l}=\lambda_{s}^{2 k+h_{k}-1}\left(T \theta_{k} K^{2 k-1} \Psi\right)_{j s}=\lambda_{s}^{2 k+h_{k}-1}\left(\theta_{k}^{(0)}\left(K^{(0)}\right)^{2 k-1} T \Psi\right)_{j s}$.

That is

$$
\left(B_{s} T^{T}\right)_{j k}=\lambda_{s}^{2 j+h_{j}-1} \xi_{j k}(T \Psi)_{k s} .
$$

Hence

$$
B_{s} T^{T}=\left(\lambda_{s}^{2 j+h_{j}-1} \delta_{j k}\right)_{n \times n}\left(\xi_{j k}\right)_{n \times 2 n}\left((T \Psi)_{j s} \delta_{j k}\right)_{2 n \times 2 n}
$$

is of rank $n$ provided that $\Psi \in S$.

Hence $\operatorname{rank}\left(B_{s}\right)=n$ if $\Psi \in S$. From (72), we have

$$
\operatorname{rank}\left(\mathcal{M}^{(s)}\right)=\operatorname{rank}\left(\mathcal{M}^{(s-1)}\right)+n
$$

if $\Psi \in S$, which implies $\operatorname{rank}\left(\mathcal{M}^{(r)}\right)=n r$ if $\Psi \in S$. 
Finite dimensional Hamiltonian system related to Lax pair with symmetries

From (67), for given $\Psi_{0} \in S$, there exists $\varepsilon_{0}>0$ such that

$$
\left.\operatorname{rank}\left(\sum_{l=1}^{2 n}\left(W^{-1}\right)_{j l} \frac{\partial E_{p}^{(k)}\left(\theta_{k}\right)}{\partial \phi_{l \sigma}}\right)_{\substack{1 \leq k \leq n, 1 \leq p \leq r \\ 1 \leq j \leq 2 n, 1 \leq \sigma \leq r}}\right|_{\Psi=\varepsilon \Psi_{0}}=n r
$$

holds for all $\varepsilon$ with $|\varepsilon|<\varepsilon_{0}$. Equivalently, $\operatorname{rank}\left(\frac{\partial E_{p}^{(k)}\left(\theta_{k}\right)}{\partial \phi_{j \sigma}}\right) \substack{1 \leq k \leq n, 1 \leq p \leq r \\ 1 \leq j \leq 2 n, 1 \leq \sigma \leq r}_{\substack{\Psi=\varepsilon \Psi_{0} \\ 1 \leq 0}}=n r$ holds for all $\varepsilon$ with $|\varepsilon|<\varepsilon_{0}$. Because of the real analyticity, the above equality holds in a dense subset of $\mathbf{R}^{2 n \times r}$. The theorem is proved.

Summarizing the results in Theorem 2,5 and 6, we have the final theorem on the integrability.

Theorem 7 Suppose $K \in \mathcal{D}_{1}$ is diagonalizable, $f \in \mathcal{F}_{p, h}(p \geq 1)$. Suppose also that there exist $\theta_{k} \in \Theta_{h_{k}}(k=1, \cdots, n)$ such that $\left[\theta_{j}, \theta_{k}\right]=0$ for all $j, k$, and $\theta_{j} K^{2 j-1}(j=1, \cdots, n)$ are linearly independent. Then the system (29) is an integrable Hamiltonian system in Liouville sense with Hamiltonian function given by (30).

With stronger conditions on $K$, we have

Corollary 2 Suppose $K \in \mathcal{D}_{1}$ is diagonalizable and $K^{2}$ has at least $n$ distinct eigenvalues. Suppose also that $f \in \mathcal{F}_{p, h}(p \geq 1)$. Then the system (29) is an integrable Hamiltonian system in Liouville sense with Hamiltonian function given by (30).

Proof: Take $\theta_{1}=\cdots=\theta_{n}=I$ in Theorem 7 . Since $K^{2}$ has at least $n$ distinct non-zero eigenvalues, $K, K^{3}, \cdots, K^{2 n-1}$ are linearly independent. The result follows from Theorem 7 .

\section{Some examples}

In this section, we will recover some known results for certain important integrable equations from the general results in the present paper. Hereafter, we always write

$$
\pi_{j, k}^{(l)}=\sum_{\sigma=1}^{r} \lambda_{\sigma}^{l} \phi_{j \sigma} \phi_{k \sigma} .
$$

\subsection{MKdV equation}

The MKdV equation

$$
u_{t}+6 u^{2} u_{x}+u_{x x x}=0
$$

has the Lax pair

$$
\begin{aligned}
\Phi_{x}= & \lambda\left(\begin{array}{cc}
1 & 0 \\
0 & -1
\end{array}\right) \Phi+\left(\begin{array}{cc}
0 & u \\
-u & 0
\end{array}\right) \Phi \\
\Phi_{t}= & -4 \lambda^{3}\left(\begin{array}{cc}
1 & 0 \\
0 & -1
\end{array}\right) \Phi-4 \lambda^{2}\left(\begin{array}{cc}
0 & u \\
-u & 0
\end{array}\right) \Phi \\
& -2 \lambda\left(\begin{array}{cc}
u^{2} & u_{x} \\
u_{x} & -u^{2}
\end{array}\right) \Phi-\left(\begin{array}{cc}
0 & u_{x x}+2 u^{3} \\
-u_{x x}-2 u^{3} & 0
\end{array}\right) \Phi .
\end{aligned}
$$


Finite dimensional Hamiltonian system related to Lax pair with symmetries

Now $n=1, N=1, m_{1}=2, \omega_{1}=-1, W=\left(\begin{array}{cc}0 & -1 \\ 1 & 0\end{array}\right), \Omega_{1}=W$, $K=\left(\begin{array}{cc}1 & 0 \\ 0 & -1\end{array}\right)$. Then $\mathcal{D}_{0}=\left\{\left(\begin{array}{cc}0 & -c \\ c & 0\end{array}\right) \mid c \in \mathbf{R}\right\}, \mathcal{D}_{1}=\left\{\left(\begin{array}{cc}a & b \\ b & -a\end{array}\right) \mid a, b \in \mathbf{R}\right\}$, $\mathcal{D}_{0} \cap \operatorname{ker} \operatorname{ad} K=\{0\}, \Theta_{0}=\left\{ \pm I_{2 \times 2}\right\}, \Theta_{1}=\emptyset$.

Take $\kappa=-1$. Let $\Phi_{\sigma}(\sigma=1, \cdots, r)$ be column solutions of (81) with $\lambda=\lambda_{\sigma}$. By (13), the Lax matrix is

$$
\begin{aligned}
& L(\lambda)=\left(\begin{array}{cc}
1 & 0 \\
0 & -1
\end{array}\right)+\sum_{k=0}^{\infty} \lambda^{-2 k-1}\left(\begin{array}{cc}
0 & \pi_{1,1}^{(2 k)}+\pi_{2,2}^{(2 k)} \\
-\pi_{1,1}^{(2 k)}-\pi_{2,2}^{(2 k)} & 0
\end{array}\right) \\
& +\sum_{k=0}^{\infty} \lambda^{-2 k-2}\left(\begin{array}{cc}
-2 \pi_{1,2}^{(2 k+1)} & \pi_{1,1}^{(2 k+1)}-\pi_{2,2}^{(2 k+1)} \\
\pi_{1,1}^{(2 k+1)}-\pi_{2,2}^{(2 k+1)} & 2 \pi_{1,2}^{(2 k+1)}
\end{array}\right) .
\end{aligned}
$$

Take

$$
f^{x}(\tau)=\tau \in \mathcal{F}_{1,0}, \quad f^{t}(\tau)=2\left(\tau^{3}-3 \tau\right) \in \mathcal{F}_{3,0}=\mathcal{F}_{1,0},
$$

then $f^{x}(K)=K, f^{t}(K)=-4 K$. According to Theorem 1, the nonlinear constraint is

$$
u=\pi_{1,1}^{(0)}+\pi_{2,2}^{(0)} \text {. }
$$

Then

$$
u_{x}=2\left(\pi_{1,1}^{(1)}-\pi_{2,2}^{(1)}\right), \quad u_{x x}=4\left(\pi_{1,1}^{(2)}+\pi_{2,2}^{(2)}\right)+8 u \pi_{1,2}^{(1)} .
$$

Under the constraint (84), the Lax pair (81) becomes

$$
\Phi_{\sigma, x}=\left.\left(\lambda f^{x}(L(\lambda))\right)_{+}\right|_{\lambda=\lambda_{\sigma}} \Phi_{\sigma}, \quad \Phi_{\sigma, t}=\left.\left(\lambda^{3} f^{t}(L(\lambda))\right)_{+}\right|_{\lambda=\lambda_{\sigma}} \Phi_{\sigma} .
$$

Remark $1 f^{x}(\tau)=-\frac{1}{2}\left(\tau^{3}-3 \tau\right) \in \mathcal{F}_{1,0}=\mathcal{F}_{3,0}$ which is proportional to $f^{t}(\tau)$ will give the same equation as $f(\tau)=\tau$.

According to Theorem 2, the systems in (86) are Hamiltonian systems with Hamiltonian functions

$$
\begin{aligned}
H^{x}= & -\frac{1}{8} \operatorname{tr} \operatorname{Res} \lambda L(\lambda)^{2}=\frac{1}{32} \operatorname{tr} \operatorname{Res} \lambda\left(L(\lambda)^{4}-6 L(\lambda)^{2}\right)=\pi_{1,2}^{(1)}+\frac{1}{4}\left(\pi_{1,1}^{(0)}+\pi_{2,2}^{(0)}\right)^{2}, \\
H^{t}= & -\frac{1}{8} \operatorname{tr} \operatorname{Res} \lambda^{3}\left(L(\lambda)^{4}-6 L(\lambda)^{2}\right)=-4 \pi_{1,2}^{(3)}-2\left(\pi_{1,1}^{(0)}+\pi_{2,2}^{(0)}\right)\left(\pi_{1,1}^{(2)}+\pi_{2,2}^{(2)}\right) \\
& +\left(\pi_{1,1}^{(1)}-\pi_{2,2}^{(1)}\right)^{2}-2\left(\pi_{1,1}^{(0)}+\pi_{2,2}^{(0)}\right)^{2} \pi_{1,2}^{(1)}-\frac{1}{4}\left(\pi_{1,1}^{(0)}+\pi_{2,2}^{(0)}\right)^{4} .
\end{aligned}
$$

These are involutive Hamiltonian systems which are integrable. The solutions of the corresponding Hamiltonian equations satisfy the MKdV equation. Therefore, we have recovered some known results [25] from our general results. 
Finite dimensional Hamiltonian system related to Lax pair with symmetries

6.2. $2 \times 2$ real AKNS system

The $x$-part of the $2 \times 2$ real AKNS system is

$$
\Phi_{x}=\lambda\left(\begin{array}{cc}
1 & 0 \\
0 & -1
\end{array}\right) \Phi+\left(\begin{array}{ll}
0 & u \\
v & 0
\end{array}\right) \Phi .
$$

Now $n=1, N=0, W=\left(\begin{array}{cc}0 & -1 \\ 1 & 0\end{array}\right), K=\left(\begin{array}{cc}1 & 0 \\ 0 & -1\end{array}\right)$. Then $\mathcal{D}_{0}=$ $\left\{\left(\begin{array}{cc}a & b \\ c & -a\end{array}\right) \mid a, b, c \in \mathbf{R}\right\}, \mathcal{D}_{0} \cap \operatorname{ker} \operatorname{ad} K=\left\{\left(\begin{array}{cc}a \\ & -a\end{array}\right) \mid a \in \mathbf{R}\right\}, \Theta_{0}=\left\{ \pm I_{2 \times 2}\right\}$.

Take $\kappa=-1$. Let $\Phi_{\sigma}(\sigma=1, \cdots, r)$ be column solutions of (88) with $\lambda=\lambda_{\sigma}$. The Lax matrix is

$$
L(\lambda)=\left(\begin{array}{cc}
1 & 0 \\
0 & -1
\end{array}\right)+\sum_{k=0}^{\infty} \lambda^{-k-1}\left(\begin{array}{cc}
-\pi_{1,2}^{(k)} & \pi_{1,1}^{(k)} \\
-\pi_{2,2}^{(k)} & \pi_{1,2}^{(k)}
\end{array}\right) .
$$

Noticing that $\mathcal{D}_{0} \cap$ ker $\operatorname{ad} K \neq\{0\}$, we need to take $f^{x}(\tau)=-\frac{1}{2}\left(\tau^{3}-3 \tau\right) \in \mathcal{F}_{3,0}=$ $\mathcal{F}_{1,0}$ as in Theorem 3. According to Theorem 1, the nonlinear constraint is

$$
u=\pi_{1,1}^{(0)}, \quad v=-\pi_{2,2}^{(0)} .
$$

Under this constraint, the Lax pair (88) becomes

$$
\Phi_{\sigma, x}=\left.\left(\lambda f^{x}(L(\lambda))\right)_{+}\right|_{\lambda=\lambda_{\sigma}} \Phi_{\sigma} .
$$

According to Theorem 2 and Corollary 2, the system (91) is an integrable Hamiltonian system with Hamiltonian function

$$
H^{x}=\frac{1}{16} \operatorname{tr} \operatorname{Res} \lambda\left(L(\lambda)^{4}-6 L(\lambda)^{2}\right)=\pi_{1,2}^{(1)}+\frac{1}{2} \pi_{1,1}^{(0)} \pi_{2,2}^{(0)} .
$$

This is a well known result [4].

\subsection{Nonlinear Schrödinger equation}

The nonlinear Schrödinger equation, written in real form, is

$$
\begin{aligned}
& u_{t}=v_{x x}+2\left(u^{2}+v^{2}\right) v, \\
& -v_{t}=u_{x x}+2\left(u^{2}+v^{2}\right) u .
\end{aligned}
$$


Finite dimensional Hamiltonian system related to Lax pair with symmetries

Denote $I=\left(\begin{array}{ll}1 & \\ & 1\end{array}\right)$ and $J=\left(\begin{array}{ll} & -1 \\ 1 & \end{array}\right)$ which play the role of 1 and $\mathrm{i}=\sqrt{-1}$ respectively. The Lax pair in real form is

$$
\begin{aligned}
\Phi_{x}= & \lambda\left(\begin{array}{rr}
I & \\
& -I
\end{array}\right) \Phi+\left(\begin{array}{rr}
-u I+v J & u I+v J
\end{array}\right) \Phi, \\
\Phi_{t}= & -2 \lambda^{2}\left(\begin{array}{c}
J \\
-J
\end{array}\right) \Phi-2 \lambda\left(\begin{array}{rr}
-v I-u J & -v I+u J
\end{array}\right) \Phi \\
& -\left(\begin{array}{rr}
\left(u^{2}+v^{2}\right) J & -v_{x} I+u_{x} J \\
v_{x} I+u_{x} J & -\left(u^{2}+v^{2}\right) J
\end{array}\right) \Phi .
\end{aligned}
$$

Now $n=2, N=2, m_{1}=2, m_{2}=2, \omega_{1}=-1, \omega_{2}=1$,

$\Omega_{1}=W=\left(\begin{array}{cccc}0 & 0 & -1 & 0 \\ 0 & 0 & 0 & 1 \\ 1 & 0 & 0 & 0 \\ 0 & -1 & 0 & 0\end{array}\right), \quad \Omega_{2}=\left(\begin{array}{cc}\mathrm{i} J & \\ & \mathrm{i} J\end{array}\right), \quad K=\left(\begin{array}{ll}I & \\ & -I\end{array}\right)$.

Then

$$
\begin{aligned}
& \mathcal{D}_{0}=\left\{\left(\begin{array}{cc}
a J & b I+c J \\
-b I+c J & -a J
\end{array}\right) \mid a, b, c \in \mathbf{R}\right\}, \\
& \mathcal{D}_{1}=\left\{\left(\begin{array}{cc}
a I & b I+c J \\
b I-c J & -a I
\end{array}\right) \mid a, b, c \in \mathbf{R}\right\}, \\
& \mathcal{D}_{0} \cap \operatorname{ker} \operatorname{ad} K=\left\{\left(\begin{array}{c}
a J \\
-a J
\end{array}\right) \mid a \in \mathbf{R}\right\}, \\
& \Theta_{0}=\left\{ \pm I_{4 \times 4}\right\}, \quad \Theta_{1}=\left\{ \pm\left(\begin{array}{l}
J \\
J
\end{array}\right)\right\} .
\end{aligned}
$$

Take $\kappa=-1$. Let $\Phi_{\sigma}(\sigma=1, \cdots, r)$ be column solutions of $(94)$ with $\lambda=\lambda_{\sigma}$. The Lax matrix is

$$
\begin{aligned}
& L(\lambda)=\left(\begin{array}{cc}
I & \\
& -I
\end{array}\right)+\sum_{k=0}^{\infty} \lambda^{-2 k-1}\left(\begin{array}{cc}
-q_{1}^{(2 k)} J & q_{2}^{(2 k)} I+q_{3}^{(2 k)} J \\
-q_{2}^{(2 k)} I+q_{3}^{(2 k)} J & q_{1}^{(2 k)} J
\end{array}\right) \\
& +\sum_{k=0}^{\infty} \lambda^{-2 k-2}\left(\begin{array}{cc}
-q_{1}^{(2 k+1)} I & q_{2}^{(2 k+1)} I+q_{3}^{(2 k+1)} J \\
q_{2}^{(2 k+1)} I-q_{3}^{(2 k+1)} J & q_{1}^{(2 k+1)} I
\end{array}\right)
\end{aligned}
$$

where

$$
\begin{aligned}
& q_{1}^{(2 k)}=2\left(\pi_{1,4}^{(2 k)}+\pi_{2,3}^{(2 k)}\right), \quad q_{2}^{(2 k)}=\pi_{1,1}^{(2 k)}-\pi_{2,2}^{(2 k)}+\pi_{3,3}^{(2 k)}-\pi_{4,4}^{(2 k)}, \\
& q_{3}^{(2 k)}=2\left(\pi_{1,2}^{(2 k)}-\pi_{3,4}^{(2 k)}\right), \quad q_{1}^{(2 k+1)}=2\left(\pi_{1,3}^{(2 k+1)}-\pi_{2,4}^{(2 k+1)}\right), \\
& q_{2}^{(2 k+1)}=\pi_{1,1}^{(2 k+1)}-\pi_{2,2}^{(2 k+1)}-\pi_{3,3}^{(2 k+1)}+\pi_{4,4}^{(2 k+1)}, \quad q_{3}^{(2 k+1)}=2\left(\pi_{1,2}^{(2 k+1)}+\pi_{3,4}^{(2 k+1)}\right) .
\end{aligned}
$$


Finite dimensional Hamiltonian system related to Lax pair with symmetries

Denote $\theta=\left(\begin{array}{cc}J & \\ & J\end{array}\right) \in \Theta_{1}$, and let

$f^{x}(\tau)=-\frac{1}{2}\left(\tau^{3}-3 \tau\right) \in \mathcal{F}_{1,0}, \quad f^{t}(\tau)=-\frac{1}{4} \theta\left(3 \tau^{5}-10 \tau^{3}+15 \tau\right) \in \mathcal{F}_{2,1}$,

then $f^{x}(K)=K, f^{t}(K)=-2 \theta K$.

Remark 2 If we take $f^{x}(\tau)=\tau$, then $f^{x}(K)=K$, but $L_{1}$ has a non-zero projection in $\mathcal{D}_{0} \cap \operatorname{ker} \operatorname{ad} K \neq\{0\}$. To solve this problem, we use Theorem 3 to get $\zeta(\tau)=-\frac{1}{2}\left(\tau^{3}-3 \tau\right)$, which gives $f^{x}(x)$ in (99).

Remark $3 f^{x}(\tau)=\frac{1}{8}\left(3 \tau^{5}-10 \tau^{3}+15 \tau\right)=-\frac{1}{2} \theta f^{t}(\tau)$ plays the same role as $f^{x}(\tau)$ in (99) does.

According to Theorem 1, the nonlinear constraint is

$$
u=\pi_{1,1}^{(0)}-\pi_{2,2}^{(0)}+\pi_{3,3}^{(0)}-\pi_{4,4}^{(0)}, \quad v=2\left(\pi_{1,2}^{(0)}-\pi_{3,4}^{(0)}\right) .
$$

Then

$$
\begin{aligned}
& u_{x}=2\left(\pi_{1,1}^{(1)}-\pi_{2,2}^{(1)}-\pi_{3,3}^{(1)}+\pi_{4,4}^{(0)}\right)-4 v\left(\pi_{1,4}^{(0)}+\pi_{2,3}^{(0)}\right), \\
& v_{x}=4\left(\pi_{1,2}^{(1)}+\pi_{3,4}^{(1)}\right)+4 u\left(\pi_{1,4}^{(0)}+\pi_{2,3}^{(0)}\right) .
\end{aligned}
$$

Under the constraint (100), the Lax pair becomes

$$
\Phi_{\sigma, x}=\left.\left(\lambda f^{x}(L(\lambda))\right)_{+}\right|_{\lambda=\lambda_{\sigma}} \Phi_{\sigma}, \quad \Phi_{\sigma, t}=\left.\left(\lambda^{2} f^{t}(L(\lambda))\right)_{+}\right|_{\lambda=\lambda_{\sigma}} \Phi_{\sigma} .
$$

According to Theorem 2, these are Hamiltonian systems with Hamiltonian functions

$$
\begin{aligned}
H^{x}= & \frac{1}{64} \operatorname{tr} \operatorname{Res}\left(\lambda\left(L(\lambda)^{4}-6 L(\lambda)^{2}\right)\right) \\
= & -\frac{1}{128} \operatorname{tr} \operatorname{Res}\left(\lambda\left(L(\lambda)^{6}-5 L(\lambda)^{4}+15 L(\lambda)^{2}\right)\right) \\
= & \pi_{1,3}^{(1)}-\pi_{2,4}^{(1)}+\left(\pi_{1,2}^{(0)}-\pi_{3,4}^{(0)}\right)^{2}+\frac{1}{4}\left(\pi_{1,1}^{(0)}-\pi_{2,2}^{(0)}+\pi_{3,3}^{(0)}-\pi_{4,4}^{(0)}\right)^{2}, \\
H^{t}= & \frac{1}{64} \operatorname{tr} \operatorname{Res}\left(\lambda^{2} \theta\left(L(\lambda)^{6}-5 L(\lambda)^{4}+15 L(\lambda)^{2}\right)\right) \\
= & 2\left(\pi_{1,4}^{(2)}+\pi_{2,3}^{(2)}\right)+2\left(\pi_{1,2}^{(1)}+\pi_{3,4}^{(1)}\right)\left(\pi_{1,1}^{(0)}-\pi_{2,2}^{(0)}+\pi_{3,3}^{(0)}-\pi_{4,4}^{(0)}\right) \\
& -2\left(\pi_{1,2}^{(0)}-\pi_{3,4}^{(0)}\right)\left(\pi_{1,1}^{(1)}-\pi_{2,2}^{(1)}-\pi_{3,3}^{(1)}+\pi_{4,4}^{(1)}\right) \\
& +\left(\pi_{1,4}^{(0)}+\pi_{2,3}^{(0)}\right)\left(\left(\pi_{1,1}^{(0)}-\pi_{2,2}^{(0)}+\pi_{3,3}^{(0)}-\pi_{4,4}^{(0)}\right)^{2}+4\left(\pi_{1,2}^{(0)}-\pi_{3,4}^{(0)}\right)^{2}\right) .
\end{aligned}
$$

According to Theorem 7 with $\theta_{1}=I$ and $\theta_{2}=\theta$, these Hamiltonian systems are integrable in Liouville sense. The solutions of the corresponding Hamiltonian equations satisfy the nonlinear Schrödinger equation. This recovers the results in [25]. 
Finite dimensional Hamiltonian system related to Lax pair with symmetries

6.4. $u(n)$ AKNS system

Denote $I$ and $J$ as in the above subsection. The $x$ part of the $u(n)$ AKNS system is the linear system

$$
\Phi_{x}=(\lambda K+P) \Phi .
$$

Here $K=\left(a_{j} J \delta_{j k}\right)_{1 \leq j, k \leq n}, a_{j}(j=1, \cdots, n)$ are real numbers such that $a_{1}, \cdots, a_{n}$ are distinct. $P=\left(u_{j k} I+v_{j k} J\right)_{1 \leq j, k \leq n}$ with $u_{j j}=v_{j j}=0, u_{k j}=-u_{j k}, v_{k j}=v_{j k}$ $(j, k=1, \cdots, n)$.

Here we have written the $u(n)$ AKNS system in real form, which is equivalent to usual complex form.

Now $m_{1}=2, N=1, \omega_{1}=1, \Omega_{1}=W=\left(-J \delta_{j k}\right)_{1 \leq j, k \leq n}$. Then

$$
\begin{aligned}
& \mathcal{D}_{0}\left(=\mathcal{D}_{1}\right)=\left\{\left(a_{j k} I+b_{j k} J\right)_{1 \leq j, k \leq n} \mid a_{j k}, b_{j k} \in \mathbf{R},\right. \\
& \left.\quad a_{k j}=-a_{j k}, b_{k j}=b_{j k}(j, k=1, \cdots, n)\right\}, \\
& \mathcal{D}_{0} \cap \operatorname{ker} \operatorname{ad} K=\left\{\left(c_{j} J \delta_{j k}\right)_{1 \leq j, k \leq n} \mid c_{j} \in \mathbf{R}(j=1, \cdots, n)\right\}, \\
& \Theta_{0}=\left\{ \pm I_{2 n \times 2 n}\right\} .
\end{aligned}
$$

Let $f^{x}=\zeta$ where $\zeta$ is given by Theorem 3 , then $f^{x}(K)=K$.

Take $\kappa=-1$. Let $\Phi_{\sigma}(\sigma=1, \cdots, r)$ be column solutions of (104) with $\lambda=\lambda_{\sigma}$. By (13), the Lax matrix is $L(\lambda)=\left(L_{j k}(\lambda)\right)_{1 \leq j, k \leq n}$ with

$$
\begin{aligned}
& L_{j k}(\lambda)=\left(\begin{array}{cc}
0 & -a_{j} \\
a_{j} & 0
\end{array}\right) \delta_{j k} \\
& +\sum_{\sigma=1}^{N} \frac{1}{\lambda-\lambda_{\sigma}}\left(\begin{array}{cc}
\phi_{2 j-1, \sigma} \phi_{2 k, \sigma}-\phi_{2 j, \sigma} \phi_{2 k-1, \sigma} & -\phi_{2 j-1, \sigma} \phi_{2 k-1, \sigma}-\phi_{2 j, \sigma} \phi_{2 k, \sigma} \\
\phi_{2 j-1, \sigma} \phi_{2 k-1, \sigma}+\phi_{2 j, \sigma} \phi_{2 k, \sigma} & \phi_{2 j-1, \sigma} \phi_{2 k, \sigma}-\phi_{2 j, \sigma} \phi_{2 k-1, \sigma}
\end{array}\right) .
\end{aligned}
$$

By Theorem 1 and 3, the nonlinear constraint is

$$
u_{j k}=\pi_{2 j-1,2 k}^{(0)}-\pi_{2 j, 2 k-1}^{(0)}, \quad v_{j k}=\pi_{2 j-1,2 k-1}^{(0)}+\pi_{2 j, 2 k}^{(0)} \quad(j \neq k) .
$$

Under this constraint, the Lax pair becomes a system of ODEs

$$
\Phi_{\sigma, x}=\left.(\lambda \zeta(L(\lambda)))_{+}\right|_{\lambda=\lambda_{\sigma}} \Phi_{\sigma} .
$$

It is too complicated to derive the Hamiltonian functions from Theorem 2 directly. However, it can be easily integrated from (108) to get the Hamiltonian functions since the action of $\zeta$ is simply to remove the ker ad $K$ component from $\widetilde{P}$. The result is that (108) is a Hamiltonian system with Hamiltonian function

$$
\begin{aligned}
H^{x}= & \frac{1}{2} \sum_{j=1}^{n} a_{j}\left(\pi_{2 j-1,2 j-1}^{(1)}+\pi_{2 j, 2 j}^{(1)}\right) \\
& +\frac{1}{4} \sum_{\substack{j, k=1 \\
j \neq k}}^{n}\left(\pi_{2 j, 2 k-1}^{(0)}-\pi_{2 j-1,2 k}^{(0)}\right)^{2}+\frac{1}{4} \sum_{\substack{j, k=1 \\
j \neq k}}^{n}\left(\pi_{2 j-1,2 k-1}^{(0)}+\pi_{2 j, 2 k}^{(0)}\right)^{2} .
\end{aligned}
$$


Remark 4 This process is just the binary nonlinearization [9] for the $u(n)$ AKNS system [10]. In fact, for any Lax pair with unitary symmetry, the complex structure induces a natural symplectic structure. Therefore, for any finite dimensional Hamiltonian systems derived by nonlinearization method from the $u(n)$ AKNS system, their conserved integrals, $r$ matrices and the Liouville integrability are derived naturally from the results of the present paper, although the Lax matrix and the Hamiltonian functions may be derived more simply by direct computation.

Remark 5 The nonlinear Schrödinger equation is also included in the $u(2)$ AKNS system. Hence the nonlinear constraint given here is also applicable to the nonlinear Schrödinger equation [26]. However, it is different from that in Subsection 6.2 because the symplectic structure here is derived directly from the complex structure, while that in Subsection 6.2 is the standard one in $s l(2, \mathbf{C})$ which is isomorphic to $s p(1, \mathbf{C})$.

\section{5. $n$ wave equation}

The $n$-wave equation is the integrability condition of the Lax pair

$$
\Phi_{x}=(\lambda K+P) \Phi, \quad \Phi_{t}=\left(\lambda K^{\prime}+Q\right) \Phi .
$$

Here $K=\left(a_{j} J \delta_{j k}\right)_{1 \leq j, k \leq n}, K^{\prime}=\left(b_{j} J \delta_{j k}\right)_{1 \leq j, k \leq n}, a_{j}, b_{j}(j=1, \cdots, n)$ are real numbers such that $a_{1}, \cdots, a_{n}$ are distinct. $P=\left(u_{j k} I+v_{j k} J\right)_{1 \leq j, k \leq n}$ with $u_{j j}=v_{j j}=0$, $u_{k j}=-u_{j k}, v_{k j}=v_{j k}(j, k=1, \cdots, n)$. Moreover, $Q=\left(\frac{b_{j}-b_{k}}{a_{j}-a_{k}}\left(u_{j k} I+v_{j k} J\right)\right)_{1 \leq j, k \leq n}$. Then $[K, Q]=\left[K^{\prime}, P\right]$. Clearly the $n$ wave equation is a special equation in the $u(n)$ AKNS system. Hence we only need to consider the $t$-part of the Lax pair.

For the $n$ wave equation, $N, m_{1}, \omega_{1}, \Omega_{1}=W, \mathcal{D}_{0}, \Theta$, the Lax matrix $L(\lambda)$ and the nonlinear constraint (107) are the same as those in the last subsection for the $u(n)$ AKNS system.

Since $\operatorname{det}\left(\left(\sqrt{-1} a_{j}\right)^{k-1}\right)_{1 \leq j, k \leq n} \neq 0$, the linear system

$$
\sum_{k=0}^{n-1}\left(a_{j} J\right)^{n-k-1}\left(\alpha_{k} I+\beta_{k} J\right)=b_{j} J \quad(j=1, \cdots, n)
$$

has a unique real solution $\alpha_{j}, \beta_{j}(j=1, \cdots, n)$. Let

$$
\hat{f}_{j}=\left(\left(\alpha_{j} I+\beta_{j} J\right) \delta_{a b}\right)_{1 \leq a, b \leq n}, \quad \hat{f}(\tau)=\sum_{j=1}^{n} \hat{f}_{n-j} \tau^{j-1},
$$

then $\hat{f}(K)=K^{\prime}$. Let $f^{t}=\hat{f} \circ \zeta$ where $\zeta$ is given by Theorem 3 , then $f^{t}(K)=K^{\prime}$.

Under the constraint (107), the Lax pair (110) becomes two systems of ODEs

$$
\Phi_{\sigma, x}=\left.\left(\lambda f^{x}(L(\lambda))\right)_{+}\right|_{\lambda=\lambda_{\sigma}} \Phi_{\sigma}, \quad \Phi_{\sigma, t}=\left.\left(\lambda f^{t}(L(\lambda))\right)_{+}\right|_{\lambda=\lambda_{\sigma}} \Phi_{\sigma} .
$$

Expand $\widetilde{L}(\lambda)=\zeta(L(\lambda))$ as

$$
\begin{aligned}
& \widetilde{L}=K+\lambda^{-1} \widetilde{P}+o\left(\lambda^{-1}\right) \\
& \widetilde{L}^{k}=K^{k}+\lambda^{-1} \sum_{j=0}^{k-1} K^{j} \widetilde{P} K^{k-j-1}+o\left(\lambda^{-1}\right) .
\end{aligned}
$$


Finite dimensional Hamiltonian system related to Lax pair with symmetries

With the identity

$$
\frac{b_{\mu}-b_{\nu}}{a_{\mu}-a_{\nu}} I=\sum_{k=1}^{n} \sum_{j=0}^{k-2}\left(\alpha_{n-k} I+\beta_{n-k} J\right)\left(a_{\mu} J\right)^{j}\left(a_{\nu} J\right)^{k-j-2},
$$

we have

$$
\begin{aligned}
& f^{t}(L)_{\mu \nu}=\hat{f}(\widetilde{L})_{\mu \nu} \\
& =K_{\mu \nu}^{\prime}+\lambda^{-1} \sum_{k=1}^{n} \sum_{j=0}^{k-2}\left(\alpha_{n-k} I+\beta_{n-k} J\right)\left(a_{\mu} J\right)^{j} \widetilde{P}_{\mu \nu}\left(a_{\nu} J\right)^{k-j-2}+o\left(\lambda^{-1}\right) \\
& =K_{\mu \nu}^{\prime}+\frac{b_{\mu}-b_{\nu}}{a_{\mu}-a_{\nu}} \widetilde{P}_{\mu \nu} .
\end{aligned}
$$

This gives the constraint on $Q: \widetilde{Q}_{\mu \nu}=\frac{b_{\mu}-b_{\nu}}{a_{\mu}-a_{\nu}} \widetilde{P}_{\mu \nu}$.

By integration, (113) becomes Hamiltonian systems with Hamiltonian functions

$$
\begin{aligned}
H^{x}= & \frac{1}{2} \sum_{j=1}^{n} a_{j}\left(\pi_{2 j-1,2 j-1}^{(1)}+\pi_{2 j, 2 j}^{(1)}\right) \\
& +\frac{1}{4} \sum_{\substack{j, k=1 \\
j \neq k}}^{n}\left(\pi_{2 j, 2 k-1}^{(0)}-\pi_{2 j-1,2 k}^{(0)}\right)^{2}+\frac{1}{4} \sum_{\substack{j, k=1 \\
j \neq k}}^{n}\left(\pi_{2 j-1,2 k-1}^{(0)}+\pi_{2 j, 2 k}^{(0)}\right)^{2}, \\
H^{t}= & \frac{1}{2} \sum_{\substack{j=1 \\
n}}^{n} b_{j}\left(\pi_{2 j-1,2 j-1}^{(1)}+\pi_{2 j, 2 j}^{(1)}\right) \\
& +\frac{1}{4} \sum_{\substack{j, k=1 \\
j \neq k}}^{n} \frac{b_{j}-b_{k}}{a_{j}-a_{k}}\left(\pi_{2 j, 2 k-1}^{(0)}-\pi_{2 j-1,2 k}^{(0)}\right)^{2}+\frac{1}{4} \sum_{\substack{j, k=1 \\
j \neq k}}^{n} \frac{b_{j}-b_{k}}{a_{j}-a_{k}}\left(\pi_{2 j-1,2 k-1}^{(0)}+\pi_{2 j, 2 k}^{(0)}\right)^{2} .
\end{aligned}
$$

These are involutive Hamiltonian systems which are integrable in Liouville sense. Each solution of these Hamiltonian systems gives a solution of the $n$ wave equation [10].

\section{Two dimensional hyperbolic $C_{n}^{(1)}$ Toda equation}

The two dimensional hyperbolic $C_{n}^{(1)}$ Toda equation is

$$
\begin{aligned}
& u_{1, x t}=\mathrm{e}^{2 u_{1}}-\mathrm{e}^{u_{2}-u_{1}}, \quad u_{n, x t}=\mathrm{e}^{u_{n}-u_{n-1}}-\mathrm{e}^{-2 u_{n}}, \\
& u_{j, x t}=\mathrm{e}^{u_{j}-u_{j-1}}-\mathrm{e}^{u_{j+1}-u_{j}} \quad(2 \leq j \leq n-1) .
\end{aligned}
$$

It has a Lax pair

$$
\Phi_{x}=(\lambda K+P(x, t)) \Phi, \quad \Phi_{t}=\lambda^{-1} Q(x, t) \Phi
$$

where $K=\left(\delta_{j+1, k}\right)_{1 \leq j, k \leq 2 n}, P=\left(p_{j} \delta_{j k}\right)_{1 \leq j, k \leq 2 n}$ with $p_{j}=u_{j, x}$ for $j=1, \cdots, n$ and $p_{j}=-u_{2 n+1-j, x}$ for $j=n+1, \cdots, 2 n, Q=\left(q_{k} \delta_{j, k+1}\right)_{1 \leq j, k \leq 2 n}$ with $q_{k}=\mathrm{e}^{u_{k+1}-u_{k}}$ for $k=1, \cdots, n-1, q_{n}=\mathrm{e}^{-2 u_{n}}, q_{k}=\mathrm{e}^{u_{2 n+1-k}-u_{2 n-k}}$ for $k=n+1, \cdots, 2 n-1, q_{2 n}=\mathrm{e}^{2 u_{1}}$. Note that $p_{j}+p_{2 n+1-j}=0, q_{j}=q_{2 n-j}$ and $q_{1} q_{2} \cdots q_{2 n}=1$. Here we use the convention $q_{2 n+j}=q_{j}$ etc.

Written in components, (119) is

$$
\phi_{j, x}=\lambda \phi_{j+1}+p_{j} \phi_{j}, \quad \phi_{j, t}=\lambda^{-1} q_{j-1} \phi_{j-1} .
$$


Finite dimensional Hamiltonian system related to Lax pair with symmetries

(118) is equivalent to

$$
Q_{x}=[P, Q], \quad P_{t}+[K, Q]=0,
$$

or

$$
q_{k, x}=\left(p_{k+1}-p_{k}\right) q_{k}, \quad p_{k, t}=q_{k-1}-q_{k},
$$

which are equivalent to the integrability condition of (119).

Now $N=1, m_{1}=2 n, \omega_{1}=\omega=\rho^{2}$ where $\rho=\exp \left(\frac{\pi \mathrm{i}}{2 n}\right), W=$ $\left((-1)^{j} \delta_{j, 2 n+1-k}\right)_{1 \leq j, k \leq 2 n}, \Omega_{1}=\left(\rho^{-2 j+1} \delta_{j k}\right)_{1 \leq j, k \leq 2 n}$. Then

$$
\begin{aligned}
& \mathcal{D}_{k}=\left\{\left(a_{i j}\right)_{2 n \times 2 n} \mid a_{i j} \neq 0 \text { only when } j-i \equiv k \bmod 2 n,\right. \\
&\text { and satisfy } \left.(-1)^{k} a_{i, i+k}+a_{1-k-i, 1-i}=0(1 \leq i \leq 2 n)\right\}, \\
& \mathcal{D}_{0} \cap \text { ker } \operatorname{ad} K=\{0\}, \\
& \Theta_{0}=\left\{ \pm I_{2 n \times 2 n} \mid c \in \mathbf{R}\right\}, \quad \Theta_{k}=\{0\} \quad(k=1,2, \cdots, 2 n-1) .
\end{aligned}
$$

We have $P \in \mathcal{D}_{0}, Q \in \mathcal{D}_{-1}$.

Take $\kappa=\frac{1}{2 n}$, Let $\Phi_{\sigma}(\sigma=1, \cdots, r)$ be column solutions of (119) with $\lambda=\lambda_{\sigma}$. By (13), the Lax matrix is

$$
L(\lambda)=K+\frac{1}{2 n} \sum_{\alpha=1}^{2 n} \sum_{\sigma=1}^{r} \frac{\Omega^{\alpha} \Phi_{\sigma} \Phi_{\sigma}^{T}\left(\Omega^{\alpha}\right)^{T} W}{\lambda-\omega^{\alpha} \lambda_{\sigma}},
$$

whose entries are

$$
L_{j k}(\lambda)=K_{j k}+\sum_{\sigma=1}^{r} \frac{(-1)^{k-1} \lambda_{\sigma}^{\{j-k\}} \lambda^{2 n-1-\{j-k\}}}{\lambda^{2 n}-\lambda_{\sigma}^{2 n}} \phi_{j \sigma} \phi_{2 n+1-k, \sigma},
$$

where $\{k\}$ is the remainder of $k$ divided by $2 n$. Here we have used the identity

$$
\sum_{\alpha=0}^{2 n-1} \frac{\omega^{-p \alpha}}{\lambda-\omega^{\alpha} \lambda_{\sigma}}=\frac{2 n \lambda_{\sigma}^{\{p\}} \lambda^{2 n-1-\{p\}}}{\lambda^{2 n}-\lambda_{\sigma}^{2 n}} .
$$

(see Lemma 2 in [27]).

Theorem 8 Under the constraint

$$
e^{u_{j}}=\Gamma^{j-\frac{1}{2}} \gamma_{0}^{-\frac{1}{2}} \prod_{k=1}^{j-1} \gamma_{k}^{-1}
$$

where

$$
\gamma_{j}=1-(-1)^{j} \pi_{j,-j}^{(-1)}, \quad \Gamma=\prod_{k=1}^{2 n} \gamma_{k}^{\frac{1}{2 n}}
$$

the Lax pair (119) of the two dimensional hyperbolic $C_{n}^{(1)}$ Toda equation is changed to a system of $O D E s$

$\phi_{j \sigma, x}=\lambda_{\sigma} \phi_{j+1, \sigma}+(-1)^{j-1} \pi_{j, 1-j}^{(0)} \phi_{j, \sigma}, \quad \phi_{j \sigma, t}=\lambda_{\sigma}^{-1} \prod_{k=1}^{2 n} \gamma_{k}^{\frac{1}{2 n}} \gamma_{j-1}^{-1} \phi_{j-1, \sigma}$, 
Finite dimensional Hamiltonian system related to Lax pair with symmetries

or equivalently,

$\Phi_{\sigma, x}=\left.(\lambda L(\lambda))_{+}\right|_{\lambda=\lambda_{\sigma}} \Phi_{\sigma}, \quad \Phi_{\sigma, t}=\lambda_{\sigma}^{-1}\left(\frac{1}{2 n} \operatorname{tr}\left(L(0)^{2 n}\right)\right)^{\frac{1}{2 n}-1} L(0)^{2 n-1} \Phi_{\sigma}$.

These ODEs are Liouville integrable Hamiltonian systems with the Hamiltonian functions

$$
\begin{aligned}
& H^{x}=\frac{1}{2} \operatorname{tr} \operatorname{Res}\left(\lambda L^{2}(\lambda)\right)=\sum_{j=1}^{2 n}(-1)^{j} \pi_{j, 2 n+2-j}^{(1)}+\frac{1}{2} \sum_{j=1}^{2 n}\left(\pi_{j, 2 n+1-j}^{(0)}\right)^{2}, \\
& H^{t}=-n\left(\frac{1}{2 n} \operatorname{tr}\left(L(0)^{2 n}\right)\right)^{\frac{1}{2 n}}=-n \prod_{j=1}^{2 n}\left(1-(-1)^{j-1} \pi_{j,-j}^{(-1)}\right)^{\frac{1}{2 n}} .
\end{aligned}
$$

Proof: Take $f^{x}(\tau)=\tau$. According to Theorem 1, the nonlinear constraint is

$$
p_{j}=(-1)^{j-1} \pi_{j, 1-j}^{(0)} \text {. }
$$

We should mention that (127) is compatible with (132) under the relation $p_{j}=u_{j, x}$. In fact, by the definition of $\gamma_{j}$ and the constraint (132),

$$
-\frac{\gamma_{j, x}}{\gamma_{j}}=p_{j+1}-p_{j}
$$

Hence $\Gamma_{x}=0$. (This can also be obtained from (144) below.) Then from (127),

$$
u_{j, x}=-\frac{1}{2} \frac{\gamma_{0, x}}{\gamma_{0}}-\sum_{k=1}^{j-1} \frac{\gamma_{k, x}}{\gamma_{k}}=p_{j}
$$

with the relation $p_{1}+p_{0}=0$.

Under the constraint (132), the first equation of the Lax pair (119) becomes

$$
\Phi_{\sigma, x}=\left.(\lambda L(\lambda))_{+}\right|_{\lambda=\lambda_{\sigma}} \Phi_{\sigma}=\left(\lambda_{\sigma} K+\widetilde{P}\right) \Phi_{\sigma}
$$

where $\widetilde{P}=\left((-1)^{j-1} \pi_{j, 1-j}^{(0)}\right)_{1 \leq j, k \leq 2 n}$.

According to Theorem 2, this is a Hamiltonian system with Hamiltonian function $H^{x}=\frac{1}{2} \operatorname{tr} \operatorname{Res}\left(\lambda L^{2}(\lambda)\right)=\sum_{j=1}^{2 n}(-1)^{j} \pi_{j, 2 n+2-j}^{(1)}+\frac{1}{2} \sum_{j=1}^{2 n}\left(\pi_{j, 2 n+1-j}^{(0)}\right)^{2}$.

The coefficient of the second equation of the Lax pair (119) is not a polynomial of $\lambda$. Hence we can not use the above general method and should construct its nonlinear constraint and Hamiltonian function directly. Similar to (26), we have

$$
\left.\left(\Omega^{\alpha} \Phi_{\sigma} \Phi_{\sigma}^{T}\left(\Omega^{\alpha}\right)^{T} W\right)_{t}=\frac{1}{\omega^{\alpha} \lambda_{\sigma}}\left[Q, \Omega^{\alpha} \Phi_{\sigma} \Phi_{\sigma}^{T}\left(\Omega^{\alpha}\right)^{T} W\right)\right]
$$

Hence

$$
L_{t}-\frac{1}{\lambda}[Q, L]=-\frac{1}{\lambda}[Q, K-\hat{K}]
$$

where

$$
\begin{aligned}
& \hat{K}=\left(\hat{K}_{j k}\right)=\frac{1}{2 n} \sum_{\sigma=1}^{r} \sum_{\alpha=0}^{2 n-1}\left(\omega^{\alpha} \lambda_{\sigma}\right)^{-1} \Omega^{\alpha} \Phi_{\sigma} \Phi_{\sigma}^{T}\left(\Omega^{\alpha}\right)^{T} W \\
& \hat{K}_{j k}=(-1)^{j} \sum_{\sigma=1}^{r} \lambda_{\sigma}^{-1}\left(\Phi_{\sigma} \Phi_{\sigma}^{T}\right)_{j,-j} \delta_{j, k-1}=\left(1-\gamma_{j}\right) \delta_{j, k-1} .
\end{aligned}
$$


$[Q, K-\hat{K}]=0$ holds if and only if $\gamma_{j} q_{j}=\gamma_{j+1} q_{j+1}$. This is equivalent to

$$
q_{j}=\gamma_{j}^{-1} \widetilde{\Gamma}
$$

for certain function $\widetilde{\Gamma}$. However, since $q_{1} q_{2} \cdots q_{2 n}=1$, we have

$$
\widetilde{\Gamma}=\left(\prod_{k=1}^{2 n} \gamma_{k}\right)^{\frac{1}{2 n}}=\Gamma
$$

and the nonlinear constraint becomes

$$
q_{j}=\Gamma \gamma_{j}^{-1}
$$

which is equivalent to (127). Meanwhile, the second equation of the Lax pair (119) can be written as the second equation of (129).

From (125), we have

$$
(L(0))_{j k}=\delta_{j+1, k}-\sum_{\sigma=1}^{r}(-1)^{j} \lambda_{\sigma}^{-1} \phi_{j \sigma} \phi_{-j, \sigma} \delta_{j+1, k}=\gamma_{j} \delta_{j+1, k} .
$$

Hence

$$
\operatorname{tr}(L(0))^{2 n}=2 n \prod_{k=1}^{2 n} \gamma_{k}
$$

and

$$
\left(L(0)^{2 n-1}\right)_{j k}=\prod_{\substack{l=1 \\ l \neq j-1}}^{2 n} \gamma_{l} \delta_{j, k+1}
$$

With the constraint (127), the second equation of (129) can be written as the second equation of (130).

With $H^{t}$ in (131), we have

$$
\begin{aligned}
\sum_{k=1}^{2 n} \hat{W}_{j k} \frac{\partial H^{t}}{\partial \phi_{k \sigma}}=\frac{1}{2} \sum_{k, a, b=1}^{2 n}(-1)^{j} \delta_{j+k, 1}\left(\frac{1}{2 n} \operatorname{tr} L(0)^{2 n}\right)^{\frac{1}{2 n}-1}\left(L(0)^{2 n-1}\right)_{b a} \frac{\partial L(0)_{a b}}{\partial \phi_{k \sigma}} \\
=\frac{1}{2} \sum_{k, a, b=1}^{2 n} \sum_{\sigma=1}^{r}(-1)^{j-1} \delta_{j+k, 1}\left(\frac{1}{2 n} \operatorname{tr} L(0)^{2 n}\right)^{\frac{1}{2 n}-1}\left(L(0)^{2 n-1}\right)_{b a} \lambda_{\sigma}^{-1} \\
\quad \cdot(-1)^{a}\left(\delta_{a k} \phi_{-a, \sigma} \delta_{a+1, b}+\phi_{a \sigma} \delta_{-a, k} \delta_{a+1, b}\right) \\
=\frac{1}{2} \sum_{k=1}^{2 n} \sum_{\sigma=1}^{r}(-1)^{j+k-1} \lambda_{\sigma}^{-1}\left(\frac{1}{2 n} \operatorname{tr} L(0)^{2 n}\right)^{\frac{1}{2 n}-1} \\
\cdot\left(\left(L(0)^{2 n-1}\right)_{k+1, k}+\left(L(0)^{2 n-1}\right)_{1-k,-k}\right) \phi_{-k, \sigma} \delta_{j+k, 1} \\
=\lambda_{\sigma}^{-1} \frac{\left(\frac{1}{2 n} \operatorname{tr} L(0)^{2 n}\right)^{\frac{1}{2 n}}}{1-(-1)^{j-1} \pi_{j-1,1-j}^{(-1)}} \phi_{j-1, \sigma} .
\end{aligned}
$$

Hence $H^{t}$ is the Hamiltonian function of the second equation of (130).

According to Theorem 5 and 6 , the Hamiltonian systems given by both $H^{x}$ and $H^{t}$ are Liouville integrable. The theorem is proved. 
Therefore, any solution of the integrable Hamiltonian systems with Hamiltonian functions (131) gives a solution of the two dimensional hyperbolic $C_{n}^{(1)}$ Toda equation. The corresponding symplectic structure is the natural one of $C_{n}^{(1)}$. The Hamiltonian systems (131) are simpler than (with space of lower dimension) that presented in [27] where the symplectic structure is derived from the complex structure.

\section{Acknowledgements}

This work was supported by the National Basic Research Program of China (973 Program) (2007CB814800) and the Key Laboratory of Mathematics for Nonlinear Sciences of Ministry of Education of China. The author is grateful to Prof. Ruguang Zhou and Prof. Shenglin Zhu for helpful discussions.

[1] Adler M and Van Moerbeke P 2002 Toda versus Pfaff lattice and related polynomials Duke Math. J. $1121-58$

[2] Babelon O, Bernard D and Talon M 2003 Introduction to Classical Integrable Systems Cambridge: Cambridge University Press

[3] Cao C W 1988 A cubic system which generates Bargmann potential and N-gap potential Chin. Q. J. Math. 3 90-96

[4] Cao C W 1990 Nonlinearization of the Lax system for AKNS hierarchy Sci. in China A33 528-536

[5] Cao C W, Geng X G and Wu Y T 1999 From the special 2+1 Toda lattice to the KadomtsevPetviashvili equation J. Phys. A32 8059-8078

[6] Cao C W, Wu Y T and Geng X G 1999 Relation between the Kodometsev-Petviashvili equation and the confocal involutive system, J. Math. Phys. 40 3948-3970

[7] Cheng Y and Li Y S 1991 The constraint of the Kadomtsev-Petviashvili equation and its special solutions Phys. Lett. A157 22-26

[8] Konopelchenko B, Sidorenko J and Strampp W 1991 (1+1)-dimensional integrable systems as symmetry constraints of (2+1) dimensional systems Phys. Lett. A157 17-21

[9] Ma W X and Strampp W 1994 An explicit symmetry constraint for the Lax pairs and the adjoint Lax pairs of AKNS systems Phys. Lett. A185 277-286

[10] Ma W X and Zhou Z X 2001 Binary symmetry constraints of N-wave interaction equations in $1+1$ and $2+1$ dimensions J. Math. Phys. 42 4345-4382

[11] Mackay N J and McGhee W A 1993 Affine Toda solitons and automorphisms of Dynkin diagrams Intl. J. Mod. Phys. A8 2791-2807

[12] McIntosh I 1994 Global solutions of the elliptic 2D periodic Toda lattice Nonlinearity 7 85-108

[13] Mikhailov A V 1981 The reduction problem and the inverse scattering method Physica 3D 73-117

[14] Mikhailov A V, Olshanetsky M A and Perelomov A M 1981 Two-dimensional generalized Toda lattice Commun. Math. Phys. 79 473-488

[15] Nimmo J J C and Willox R 1997 Darboux transformations for the two-dimensional Toda system Proc. Roy. Soc. London A453 2497-2525

[16] Nirov K S and Razumov A V 2008 Abelian Toda solitons revisited Rev. Math. Phys. 20 1209-1248

[17] Qiao Z J, Cao C W and Strampp W 2003 Category of nonlinear evolution equations, algebraic structure, and r-matrix J. Math. Phys. 44 701-722

[18] Qin Z Y, Zhou Z X and Zhou R G 2008 Integrable Hamiltonian systems related to the AKNS system with matrix potentials Mod. Phys. Lett. B22 2831-2842

[19] Ragnisco O, Cao C W and Wu Y T 1995 On the relation of the stationary Toda equation and the symplectic maps J. Phys. 28 573-588

[20] Ragnisco O and Rauch-Wojciechowski S 1992 Restricted flows of the AKNS hierarchy Inverse Problems 8 245-262 
[21] Ragnisco O and Rauch-Wojciechowski S 1996 Integrable maps for the Garnier and for the Neumann system J. Phys. A29 1115-1124

[22] Rogers C and Schief W K 2000 Bäcklund and Darboux Transformations, Geometry and Modern Applications in Soliton Theory Cambridge: Cambridge University Press

[23] Terng C L 2008 Geometries and symmetries of soliton equations and integrable elliptic equations Surveys on Geometry and Integrable Systems (Guest M, Miyaoka R and Ohnita Y Ed.) Advanced Studies in Pure Mathematics (Singapore: World Scientific) 51 401-488

[24] Zeng Y B 2000 Deriving N-soliton solutions via constrained flows J. Phys. A33 L115CL120

[25] Zhou R G 2007 Nonlinearizations of spectral problems of the nonlinear Schrödinger equation and the real-valued modified Korteweg-de Vries equation J. Math. Phys. 48013510

[26] Zhou R G 2009 Finite-dimensional integrable Hamiltonian systems related to the nonlinear Schrödinger equation Studies in Appl. Math. 123 311-335

[27] Zhou Z X 2007 Finite dimensional integrable systems related to two dimensional $A_{2 l}^{(2)}, C_{l}^{(1)}$ and $D_{l+1}^{(2)}$ Toda equations J. Geom. Phys. 57 1037-1053

[28] Zhou Z X 2008 Darboux transformations of lower degree for two dimensional $C_{l}^{(1)}$ and $D_{l+1}^{(2)}$ Toda equations Inverse Problems 24045016

[29] Zhou Z X 2009 The relationship between the hyperbolic Nizhnik-Novikov-Veselov equation and the stationary Davey-Stewartson II equation Inverse Problems $\mathbf{2 5} 025003$

[30] Zhou Z X, Ma W X and Zhou R G 2001 A finite-dimensional integrable system associated with Davey-Stewartson I equation Nonlinearity 14 701-717 